\title{
Numerical study of self-induced transonic flow oscillations behind a sudden duct enlargement
}

\author{
Thomas Emmert, ${ }^{1}$ Philippe Lafon, ${ }^{1}$ and Christophe Bailly ${ }^{2, a)}$ \\ ${ }^{1}$ Laboratoire de Mécanique des Structures Industrielles Durables, UMR CNRS EDF 2832, \\ Clamart 92141, France \\ ${ }^{2}$ Laboratoire de Mécanique des Fluides et d'Acoustique, École Centrale de Lyon \\ and UMR CNRS 5509, Ecully 69134, France and Institut Universitaire de France, \\ Paris 75005, France
}

(Received 19 February 2009; accepted 8 September 2009; published online 28 October 2009)

\begin{abstract}
A sonic flow in a plane duct passing an abrupt increase in cross section is studied using compressible large-eddy simulations. Different flow patterns are likely to appear in this configuration according to the ratio between the downstream ambient pressure and the upstream reservoir pressure. For low pressure ratios, the flow is entirely supersonic in the channel and a steady symmetrical shock pattern is observed. For higher pressure ratios, the flow can be attached to one side of the channel with a jet-like shock cell structure, or can be characterized by strong oscillations of a single normal shock located near the sudden expansion, known as base-pressure oscillations in literature. A hysteresis phenomenon is found experimentally and the state reached by the transonic flow depends on the path followed by the pressure ratio. Moreover, a coupling of these base-pressure oscillations with the quarter-wavelength resonance of the duct can occur. All these regimes are numerically investigated and the results are favorably compared to available experimental data. A case of frequency locking of this self-excited mechanism is also reproduced, in agreement with a modeling of the resonator. The governing equations are solved using high-order central finite differences combined with an overset grid approach. The large-eddy simulations are based on a relaxation filtering and a nonlinear shock-capturing scheme is also implemented for shock waves. (C) 2009 American Institute of Physics. [doi:10.1063/1.3247158]
\end{abstract}

\section{INTRODUCTION}

Shock-induced flow oscillations behind a sudden enlargement of cross section are frequently generated by pressure-reducing valves and by flow control devices in pipe systems of power plants. Large pressure oscillations are however quite undesirable to prevent excitation of structural vibrations and fatigue. This problem is also representative of transonic and supersonic flows involving unstable shock patterns and yielding increased fluctuating pressure amplitudes, as discussed in the review by Meier et al. ${ }^{1}$ In the present work, the studied configuration is displayed in Fig. 1. Air at atmospheric conditions, denoted by subscript $a$, is drawn through a convergent nozzle of height $h$ and is abruptly expanded in the main rectangular channel of larger height $H$ and of length $L$. For a given geometry, the behavior of the flow is driven by the exit pressure $p_{e}$ in the plenum chamber downstream of the expansion duct or equivalently by the pressure ratio $\tau=p_{e} / p_{a}$, where $p_{a}$ is the stagnation pressure in the upstream reservoir. This flow has been investigated experimentally for different values of the area ratio $h / H$ and of the channel length-to-height ratio $L / H$. Boundary layershock wave interactions, flow separations, as well as basepressure oscillations, hysteresis phenomena, and coupling with an acoustic duct resonance are reported by Anderson et $a .^{2}$ and by Meier et $a .^{3,4}$

\footnotetext{
a) Author to whom correspondence should be addressed. Electronic mail: christophe.bailly@ec-lyon.fr.
}

As an illustration of the different flow regimes that can occur in such a configuration, the evolution of the normalized mean base pressure $p_{w} / p_{a}$ at the bottom and top corner region is shown in Fig. 2 as a function of the pressure ratio $\tau$ for a given duct geometry. Three different flow regimes can roughly be identified. For lower values of the pressure ratio, $\tau \leq 0.25$, the mean base pressure $p_{w}$ is nearly constant and the flow inside the duct is entirely supersonic and symmetrical. This steady flow pattern consists of reflected oblique shock waves interacting with the wall boundary layers. This regime can be illustrated by the interferograms provided by Meier et al. ${ }^{3}$ Figure 3(a) corresponds typically to this first regime, which will be denoted regime $\mathrm{A}$ in what follows.

Note that the experimental points of Fig. 2 are not directly associated with the visualizations of Fig. 3 obtained for a slightly different geometry. The two geometrical configurations are however studied in the present work, and the visualizations are not always available in the considerable work provided by the authors. ${ }^{3}$ Increasing the plenumchamber pressure $p_{e}$, and thus the pressure ratio $\tau=p_{e} / p_{a}$, where $p_{a}$ is the reservoir pressure, leads to a contraction and eventually to a breakdown of the periodic shock-cell structure.

For higher pressure ratios in the range $0.300 \leq \tau$ $\leq 0.352$, a normal shock can occur at the center of the channel, as illustrated in Fig. 3(b). The flow is then subsonic behind the shock, and also upstream of the shock between the core flow boundaries and the walls. As a result, the cor- 


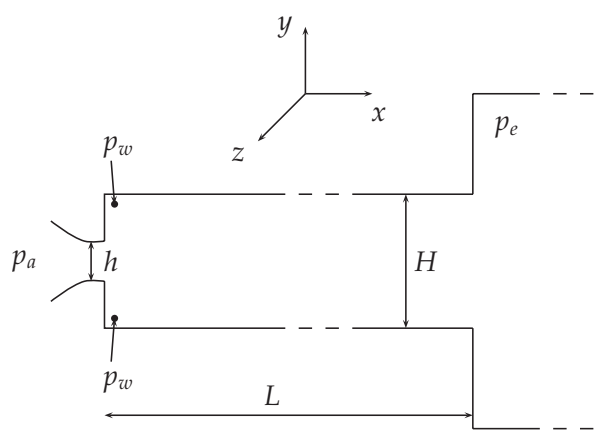

FIG. 1. Sudden enlargement of rectangular duct cross section: The flow regime is characterized by the pressure ratio between the plenum chamber and the upstream ambient pressure $\tau=p_{e} / p_{a}$. Note that the flow is supersonic for $\tau<[2 /(\gamma+1)]^{\gamma /(\gamma-1)} \simeq 0.53$, where $\gamma=1.4$ is the specific heat ratio. The cross-sectional ratio is given by $h / H$, where $h$ is the nozzle height and $H$ is the test-duct height, $L$ is the duct length, and $b$ is the width of the nozzle and of the duct, in the spanwise direction. The flow can be monitored by the base pressure $p_{w}$ at the two upstream corners of the channel.

ner regions are no longer dead zones and large amplitude perturbations can occur, namely, base pressure oscillations. An unsteady symmetrical flow pattern is then observed for this regime $\mathrm{B}$ and the snapshot is taken during the oscillation cycle. For pressure ratios $0.316 \leq \tau \leq 0.352$, the flow can also be attached either to the top or bottom wall of the channel without preference, as shown in Fig. 3(c). As a result, two values of the base pressures are reported in Fig. 2, the lower value of $p_{w}$ being associated with the attached side. In this regime $\mathrm{C}$, the flow pattern has a more jet-like structure and no oscillating mechanism similar to the normal shock configuration of regime $\mathrm{B}$ is observed for the configurations considered in this work.

To summarize, three different mean base pressure values are reported in this range of pressure ratio, one for regime $\mathrm{B}$ and two for regime C. Furthermore, the state taken by the flow, namely, oscillating symmetrical regime B or asymmetrical regime $\mathrm{C}$, depends on whether the flow is driven with an increasing or a decreasing downstream pressure $p_{e}$. Experimentally, the symmetrical oscillating flow pattern is kept for an increasing pressure ratio until $\tau \simeq 0.352$ and then lost in favor of an asymmetrical flow pattern. When the pres-

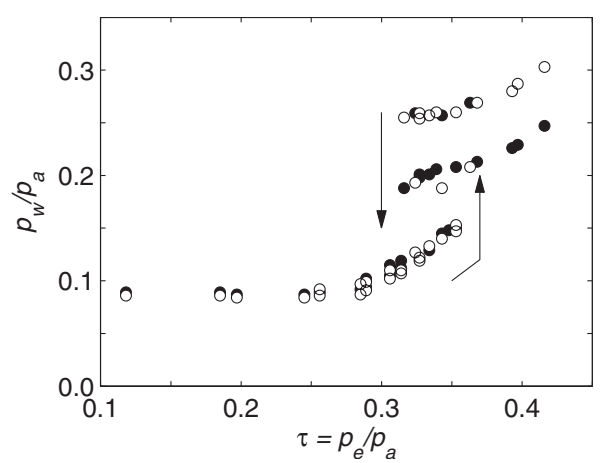

FIG. 2. Turbulent flow behind a sudden enlargement of rectangular duct cross section for the case $L=0.16 \mathrm{~m}, L / H=4.82$, and $h / H=0.3$. Experimental data (Ref. 2) of the $(\bigcirc)$ upper and (O) lower normalized mean base pressure $p_{w} / p_{a}$ as a function of the pressure ratio $\tau$.

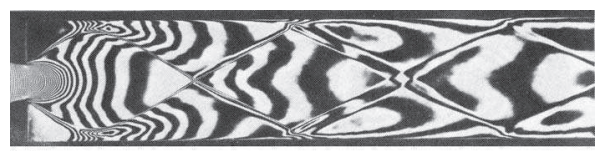

(a)

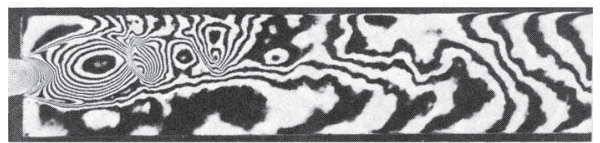

(b)

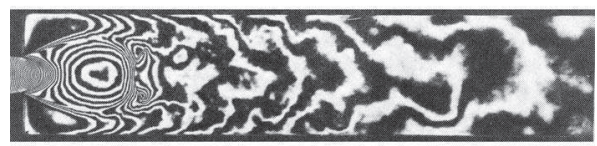

(c)

FIG. 3. Mach-Zehnder interferometry visualizations of the flow, i.e., fringes represent lines of constant density, for different pressure ratios: (a) $\tau$ $=0.151$ symmetrical supersonic flow, denoted regime A; (b) $\tau=0.364$ symmetrical flow with a normal shock, denoted regime B; (c) $\tau=0.377$ asymmetrical flow with jetlike shock cells, denoted regime $\mathrm{C}$. The expansion duct length is $L=0.24 \mathrm{~m}$ and the aspect ratios are $L / H=7.23$ and $h / H=0.3$, taken from Ref. 3.

sure ratio decreases the asymmetrical flow pattern switches to the oscillating flow pattern at $\tau \simeq 0.316$. This hysteresis loop is indicated by two arrows in Fig. 2.

Furthermore, the self-exciting mechanism observed for the symmetrical flow pattern of regime B induces weak and irregular low-frequency shock oscillations, and the frequency can be locked by the longitudinal acoustic resonances of the channel. A strong coupling is then found between the shock motion and the resonant duct. Induced high fluctuating pressure levels can result in possible damage to the whole pipe system in practice.

This flow physics involving turbulence, shocks, interactions with boundary layers, and aeroacoustic resonances is a real challenge for computational aeroacoustics. ${ }^{5,6}$ Meier et $a l .{ }^{3}$ provided time sequence visualizations of the different flow regimes based on the Mach-Zehnder interferometry. Static wall pressure data and frequency spectra of the pressure fluctuations along the walls are also available, making it possible to perform a quantitative validation of the numerical simulations. In the present work, a numerical solver of the compressible Navier-Stokes equations called Code_Safari (for simulation of aeroacoustic flows and resonance and interaction) has been developed to simulate aeroacoustic couplings for internal flows. High-order schemes are used to preserve the generated acoustic field and a nonlinear adaptive filter is implemented to correctly capture strong shock waves. Moreover, a high-order overset grid ability has been adapted in the code in order to handle complex geometries and flow regions with disparate characteristic scales. Note that the numerical algorithm must be sufficiently robust in order to treat shocks and supersonic wall flows, and also accurate enough to retain sound waves that result from complex nonlinear interactions. A previous study ${ }^{7}$ has shown that an overly high-dissipative numerical approach and a twodimensional (2D) geometry are not able to capture the coupling between the shock oscillations and the resonant duct. 
TABLE I. Pressure ratio and geometrical parameters of the computed configurations.

\begin{tabular}{lccccccc}
\hline \hline Case & $\tau$ & $L(\mathrm{~m})$ & $L / H$ & $h / H$ & $b / H$ & Regime & Flow feature \\
\hline R1 & 0.15 & 0.24 & 7.23 & 0.3 & 3.01 & A & Steady symmetrical \\
R2 & 0.30 & 0.16 & 4.82 & 0.3 & 3.01 & B & Base-pressure oscillations \\
R3 & 0.31 & $\ldots$ & $\cdots$ & $\ldots$ & 3.01 & B & Base-pressure oscillations \\
R4 & 0.32 & $\ldots$ & $\ldots$ & $\ldots$ & 3.01 & C & Asymmetrical flow pattern \\
R5 & 0.348 & $\ldots$ & $\ldots$ & $\ldots$ & 3.01 & C & Asymmetrical flow pattern \\
R6 & 0.41 & 0.08 & 5.33 & 0.4 & 6.67 & B & Acoustic resonance \\
\hline \hline
\end{tabular}

The paper is organized as follows. The numerical algorithm is first briefly discussed in Sec. II. In Sec. III, a low pressure ratio $\tau=0.15$ case corresponding to regime $\mathrm{A}$ is presented. Regimes B and C are investigated in Sec. IV for different pressure ratios, $0.30 \leq \tau \leq 0.35$, associated with the hysteresis cycle shown in Fig. 2. A coupling of the basepressure oscillations with the first longitudinal acoustic mode of the channel, leading to a frequency selection of the selfexcited shock oscillations, is presented in Sec. V. The geometry of the different studied configurations is provided in Table I. Concluding remarks are finally drawn in Sec. VI. The formulation of the shock detection used in the adaptive nonlinear filtering and the derivation of an analytical expression of the base pressure are reported in Appendixes A and $\mathrm{B}$, respectively.

\section{NUMERICAL STRATEGY}

The compressible three-dimensional (3D) Navier-Stokes equations written in conservative form after application of a general time-invariant curvilinear coordinate transformation from physical space to computational space, $(x, y, z)$ $\rightarrow(\xi, \eta, \zeta)$ are solved. This yields

$$
\frac{\partial}{\partial t}\left(\frac{\mathbf{Q}}{J}\right)+\frac{\partial \mathbf{E}}{\partial \xi}+\frac{\partial \mathbf{F}}{\partial \eta}+\frac{\partial \mathbf{G}}{\partial \zeta}=0,
$$

where $J$ is the Jacobian of the geometric transformation. The unknown vector in the above equation writes $\mathbf{Q}$ $=\left(\rho, \rho u, \rho v, \rho w, \rho e_{t}\right)^{T}$, where $\rho$ designates the density, $u, v, w$ the Cartesian velocity components, and $e_{t}$ the total specific energy, given by $\rho e_{t}=p /(\gamma-1)+\rho\left(u^{2}+v^{2}+w^{2}\right) / 2$ for a perfect gas. The flux vectors $\mathbf{E}, \mathbf{F}$, and $\mathbf{G}$ contain the inviscid and the viscous terms. Their expressions as well as the metric identities for the grid transformation can be found in the works of Visbal and Gaitonde ${ }^{8}$ or Marsden et al. ${ }^{9}$ The molecular dynamic viscosity is classically calculated by using Sutherland's law.

For interior points of the computational domain, the fluxes and the velocity derivatives involved in the viscous terms are calculated by the centered 11-point finite difference scheme developed by Bogey and Bailly. ${ }^{10}$ This scheme has been optimized in wavenumber space and is able to accurately differentiate perturbations discretized by at least four points per wavelength. The time integration is performed by an explicit fourth-order low-storage Runge-Kutta scheme. The Courant-Friedrichs-Lewy (CFL) number is 0.7 and the time step $\Delta t$ is updated every iteration during the transient phase. An optimized explicit 11-point low pass filter removes grid-to-grid oscillations, not resolved by centered finite difference schemes. ${ }^{10,11}$ The large-eddy simulation is based on a relaxation filtering to properly remove the nonresolved turbulent structures by draining the cascade energy near the grid cutoff frequency. This method was successfully applied by Bogey and Bailly ${ }^{12,13}$ for round subsonic jets, by Berland et $a l .{ }^{14}$ for computing screech noise of a supersonic jet, or more recently by Marsden et al. ${ }^{15}$ for airfoil noise using curvilinear coordinates. The filtering coefficient is chosen to be 0.2 inside the computational domain. In regions with strong gradients, an additional shock-capturing procedure is introduced, and the adaptive nonlinear artificial dissipation model proposed by Kim and Lee ${ }^{16}$ is used in the present work. The standard Jameson shock sensor has been modified and more details are provided in Appendix A.

The previous finite-difference schemes are designed for structured grids. In order to treat more complex geometries, a high-order overset ability has been adapted and implemented in the solver. In this approach, the computational domain is subdivided into overlapping structured grid components. The governing equations are solved on each component grid separately and domain connectivity is obtained through the use of interpolation polynomials. This Chimera grid method was first proposed by Benek et al. ${ }^{17}$ and extended for aeroacoustic simulations by Delfs. ${ }^{18}$ The grid assembler module ogen of the freely available library Overture developed at the Lawrence Livermore National Laboratory has been interfaced with the solver. The conception of Overture as a library makes it easy to call grid assembler functions during computation. Fluid-structure interaction problems involving relative motion of multiple bodies can also be simulated. ${ }^{19}$ For communication between grid boundaries that do not coincide, a high-order interpolation is used. Lagrangian polynomials were found by Sherer and $\mathrm{Scott}^{20}$ to be best suited in terms of accuracy, execution time, and implementation aspects for the high-order overset grid approach. Various tests have shown that at least eighth-order polynomials have to be used in order to make the error of the interpolation negligible when using the 11-point finite difference scheme. For load balancing purpose, each component grid is subdivided evenly $N$ times in each direction and can thus be computed by $N_{\text {procs }}=N_{\xi \text {,procs }} \times N_{\eta \text {,procs }} \times N_{\zeta \text {,procs. }}$. In a preprocessing step, the algorithm distributes the data concerning the computational grid and the interpolation provided by ogen for the parallel computation. The standard Message Passing Interface library routines have been used for code parallelization.

Different grids have been used in this study. The com- 

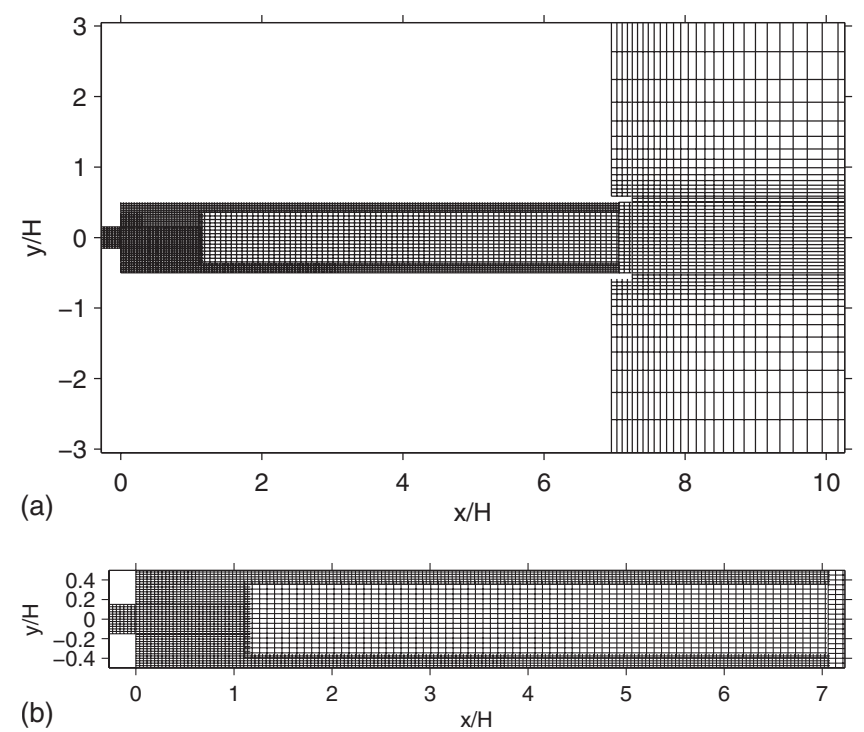

FIG. 4. Grid generated by using ogen for case R1 corresponding to a pressure ratio $\tau=0.15$; see Table I. Every eighth line is represented in the $x-y$ plane. (a) Entire computational domain including the nozzle, the expansion duct, and the plenum chamber. (b) Detailed view on the nozzle and of the expansion duct. The walls are refined using overlapping grids.

mon features are recalled in this section whereas specific mesh statistics are given in Secs. III-V. The entire overset grid generated by using ogen is represented in Fig. 4(a), and is built up in three parts: the nozzle, the expansion duct, and the reservoir. The grid points in the nozzle and in the expansion duct are spaced uniformly in each direction. The reservoir grid is stretched in the $x$-direction on the last 30 points and in the $y$-direction on the last 50 points with a ratios of $3 \%$ and $1 \%$, respectively. The grid in the spanwise $z$-direction is uniformly spaced. To describe the interaction between the boundary layers developing along the walls of the expansion duct and the shocks, a finer grid resolution is required in these regions. This can be accomplished by the overset grid approach that allows to patch grids of arbitrarily refinement in the regions of interest, as shown in Fig. 4(b). Refined meshes have been used for the nozzle and for the near wall region of the expansion channel. Periodic boundary conditions are used in spanwise direction.

The values of pressure and of temperature of air at rest in the upstream room, which is not included in the present simulation, are provided by Meier et al., ${ }^{3} \quad p_{a}=1.01$ $\times 10^{5} \mathrm{~Pa}$ and $T_{a}=293 \mathrm{~K}$. The convergent part of the nozzle is also not included in the computational domain, and the critical inflow conditions are thus computed using isentropic relations for a sonic flow, which yields

$$
\begin{aligned}
& M_{\text {in }}=u_{\text {in }} / c_{\text {in }}=1.01, \quad v_{\text {in }}=0, \quad w_{\text {in }}=0, \\
& p_{\text {in }}=0.52 p_{a}, \quad T_{\text {in }}=0.83 T_{a} .
\end{aligned}
$$

The incoming boundary layer was not documented by the authors, ${ }^{3}$ but the nozzle was designed to produce a thin boundary layer at the exit. Therefore, the velocity profile at the nozzle inflow is kept uniform in the present simulations. When no grid refinement is used near the channel walls, namely, cases R2-R6, slip conditions are applied only along

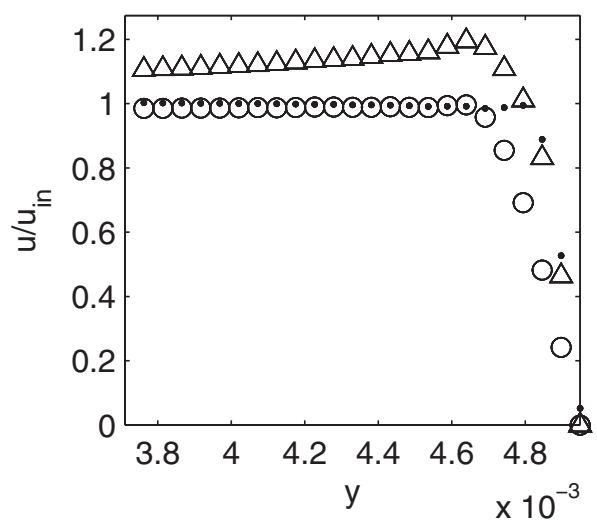

FIG. 5. Velocity profiles of the incoming laminar boundary layer at three locations: $(\bigcirc)$ at $x=-L_{n},(\bigcirc)$ at $x=-L_{n} / 2$, and $(\triangle)$ at $x=0$, where $L_{n}=9.1$ $\times 10^{-3} \mathrm{~m}$ is the nozzle length.

the nozzle wall. This ensures that the boundary layer remains sufficiently thin at the nozzle outflow. When grid refinement is used, namely, for case R1, adiabatic no-slip conditions are applied along the nozzle and a laminar velocity profile develops along the nozzle walls. Velocity profiles at three positions in the $x$-direction of the nozzle are reported in Fig. 5. The thickness of the boundary layer is around $\Delta \simeq 0.4$ $\times 10^{-3} \mathrm{~m}$ and the developing boundary layer is discretized by eight points over the fine grid.

Adiabatic no-slip boundary conditions are applied along all the walls of the expansion duct. As mentioned before, the pressure in the plenum chamber $p_{e}$ is fixed by the pressure ratio $\tau$ and the temperature is imposed at $T_{e}=T_{a}=293 \mathrm{~K}$. The nonreflective boundary conditions of Tam and Dong ${ }^{21}$ extended to 3D geometries by Bogey and Bailly ${ }^{22}$ are used at the reservoir boundaries. A sponge zone is also classically implemented in order that turbulent perturbations leave the computational domain without spurious acoustic reflections. $^{22}$

\section{SYMMETRICAL STEADY SUPERSONIC FLOW PATTERN}

In this section, a low pressure ratio of $\tau=0.15$ associated with the geometrical parameters reported in Table I, denoted case R1 hereafter, is first studied. The turbulent flow is organized by a steady shock-cell structure, as shown in Fig. 3 for regime $\mathrm{A}$. The Reynolds number based on the nozzle height $h$ and the inflow velocity $u_{\text {in }}$ is $\operatorname{Re}_{h}=2.1 \times 10^{5}$. Details about number of grid points and grid cell sizes are given in Table II. A total number of $43 \times 10^{6}$ grid points has been distributed over $N_{\text {procs }}=258$, and around $13 \times 10^{6}$ iterations have been performed in about 310 hours on a Linux cluster.

\section{A. Mean flow field}

An interferogram-like plot showing the computed mean density field is represented in Fig. 6(a) and can be compared to the view (b) observed by Meier et al. ${ }^{3}$ for the same geometry. The two pictures are qualitatively in good agreement. A divergent supersonic jet formed by the expansion waves generated at the nozzle edges is visible. This flow pattern can be found for not perfectly expanded supersonic jets since the jet 
TABLE II. Characteristics of the overset mesh for the low pressure ratio $\tau=0.15$, case R1. The length scales are given in wall units, $y^{+}=y u_{f} / \nu_{w}$, the wall-friction velocity $u_{f} \simeq 9.4 \mathrm{~m} \mathrm{~s}^{-1}$ is computed at the location $x / H=3.5$, and $\nu_{w} \simeq 6.9 \times 10^{-5} \mathrm{~m}^{2} \mathrm{~s}^{-1}$ denotes the value of the viscosity at the wall.

\begin{tabular}{lrrrrrrc}
\hline \hline & \multicolumn{1}{c}{$N_{x}$} & $N_{y}$ & $N_{z}$ & $\Delta x_{\min }^{+}$ & $\Delta y_{\min }^{+}$ & $\Delta z_{\min }^{+}$ & $N_{\text {procs }}$ \\
\hline Nozzle & 116 & 193 & 41 & 10.5 & 7.0 & 42.1 & 6 \\
Expansion duct (central) & 706 & 109 & 41 & 42.1 & 28.1 & 42.1 & 15 \\
Expansion duct (refined region) & 285 & 399 & 41 & 10.5 & 7.0 & 42.1 & 31 \\
Lower near wall grid & 3052 & 121 & 41 & 10.5 & 7.0 & 42.1 & 86 \\
Upper near wall grid & 3052 & 121 & 41 & 10.5 & 7.0 & 42.1 & 86 \\
Reservoir & 292 & 341 & 41 & 42.1 & 28.1 & 42.1 & 34 \\
\hline \hline
\end{tabular}

exit pressure $p_{\text {in }}$ is greater than the pressure $p_{e}$ imposed at the channel exit. Assuming a quasi-one-dimensional isentropic flow, the equivalent Mach number $M_{j}$ of the corresponding perfectly expanded jet

$$
M_{j}=\sqrt{\frac{2}{\gamma-1}\left(\tau^{-(\gamma-1) / \gamma}-1\right)}
$$

would be $M_{j} \simeq 1.90$. The pressure in the corner regions does not match the pressure of the expanding supersonic jet. The mismatch is compensated by a compression near the nozzle edges. The first oblique shock wave appears when the expansion waves are reflected by the upper and lower wall, and the axial maximum Mach number $M=3.7$ is then attained in the simulation. Further downstream, the shock waves are reflected on the lower and upper wall, respectively, and form a symmetrical cell structure. However, the density maximum observed downstream of the jet reattachment is less extended in the computation. A zoom on this zone is shown in Fig. 7. This density maximum is slightly detached from the wall indicating more complex interactions in the presence of the boundary layer. This specific point is discussed in Sec. III B.

The computed and measured static mean pressures along the lower wall are displayed in Fig. 8. Numerical results and experiments are in reasonable agreement. The first pressure peak caused by the jet reattachment is accurately predicted even though the peak is too narrow. The subsequent expansion fits correctly with the experimental pressure curve. A second compression indicating the reflection of the oblique shock is also well predicted in its amplitude but is located slightly further downstream.

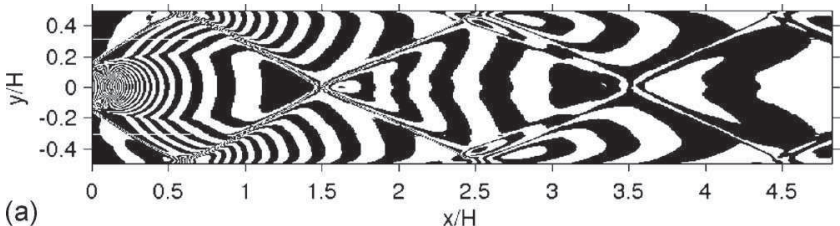

(b)

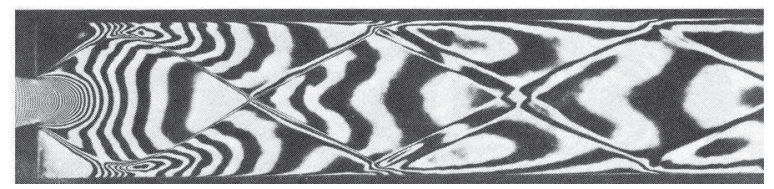

FIG. 6. Pressure ratio $\tau=0.15$, case R1. Mean density field using interferogram-like color map. (a) Present computation, 60 isocontours between 0 and $0.742 \mathrm{~kg} \mathrm{~m}^{-3}$; (b) experiments (Ref. 3).

\section{B. Some aspects of the turbulent flow}

A supersonic boundary layer develops along the duct walls downstream of the jet reattachment and interacts with the impinging oblique shock waves. An instantaneous numerical Schlieren visualization of the entire computational domain and a zoom in the jet reattachment region are shown in Figs. 9(a) and 9(b), respectively. The expanding jet is bounded by a contact discontinuity and a thin shear layer. Small compression waves are emitted from instabilities developing and growing in this thin shear layer. Its impingement on the wall enhances turbulent mixing and shifts the reattachment zone. Behind the jet reattachment zone, coherent structures that are shed at lower frequencies can be observed and might be linked to development of instability waves in the free shear layer further upstream. It is well known that the amplification of turbulence and the change in scales are the two main consequences of the interaction with shocks. $^{23}$

Visualization of the first and second oblique shock including the boundary layer interaction are displayed in Figs. 9 (c) and $9(\mathrm{~d}) .^{24}$ The incoming boundary layer seems to be transitional. Shock wave-boundary layer interactions are observed and the downstream boundary layer is more turbulent. This mixing enhancement is typical for such interactions. ${ }^{25}$ Figure 9(e) shows the shock wave-boundary layer interaction near the outflow. A normal shock pattern is observed with a lambda structure near the wall. The boundary layer separates at the vicinity of the upstream foot leg and turbulent mixing is again enhanced.

From a numerical point of view, no spurious oscillations are observed when the oblique shocks cross the interpolation zone between two overset grids. The background lowdissipation filtering combined with the nonlinear filter ap-

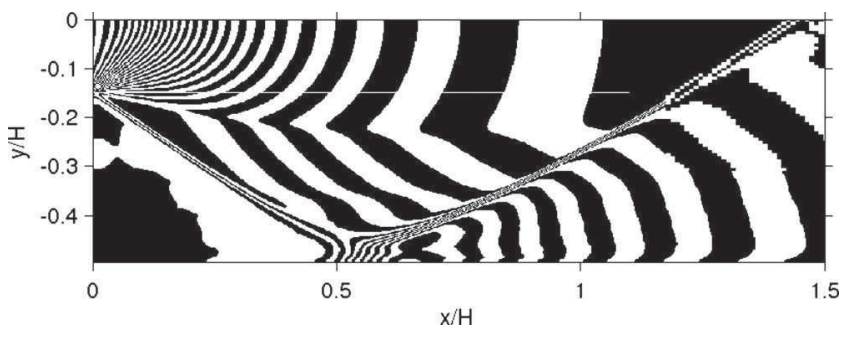

FIG. 7. Pressure ratio $\tau=0.15$, case R1. Zoom on the reattachment zone of the abruptly expanded supersonic jet; refer to Fig. 6. 


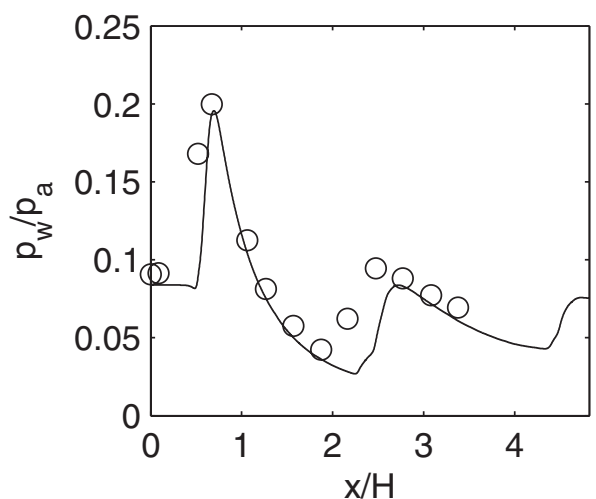

FIG. 8. $\tau=0.15$, case R1. Static mean pressure distribution along the duct wall and normalized by $p_{a} ;(-)$ present computation; $(\bigcirc)$ experiments (Ref. $3)$.
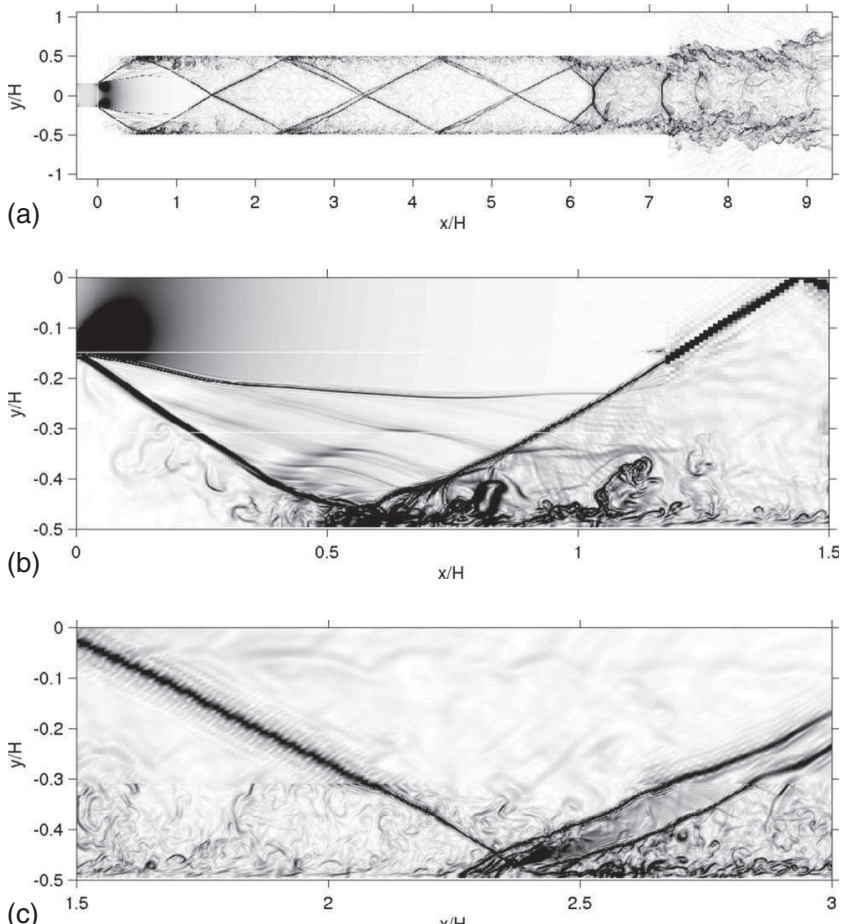

(c)
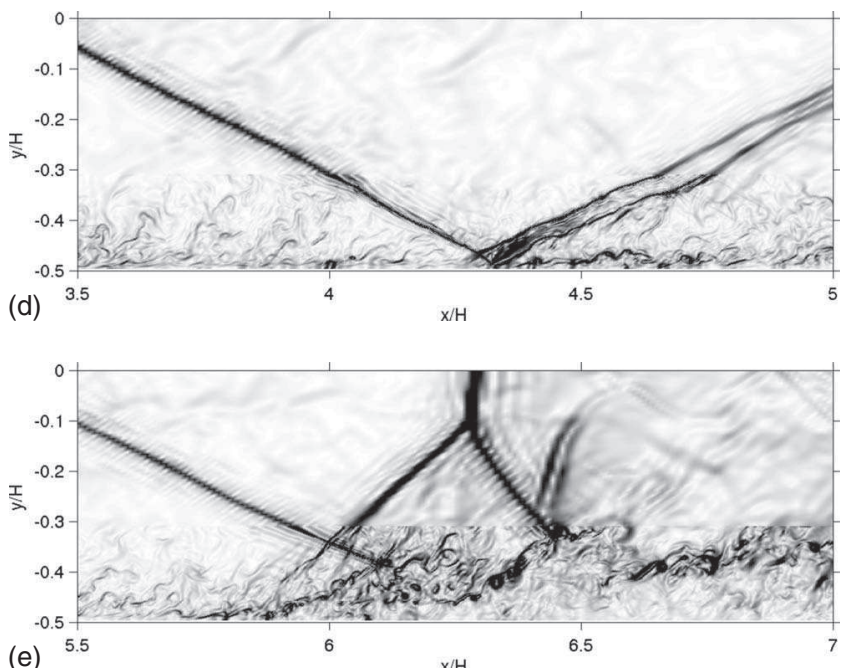

(e) ${ }^{5}$

$\mathrm{x} / \mathrm{H}$

FIG. 9. Pressure ratio $\tau=0.15$, case R1. Instantaneous numerical Schlieren field, $0 \leq|\nabla \rho| \leq 60 \mathrm{~kg} \mathrm{~m}^{-4}$. (a) Entire computational domain; (b) jet reattachment region; (c) first shock wave/boundary layer interaction zone; (d) second shock wave/boundary layer interaction zone; (e) third shock wave/ boundary layer interaction zone with flow separation near the end of the duct, at $x / H=7.23$.
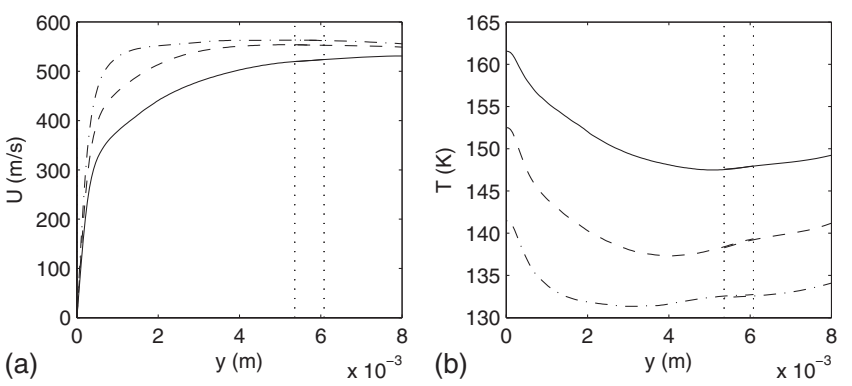

FIG. 10. Pressure ratio $\tau=0.15$, case R1. Profiles of the mean streamwise velocity $U$ (a) and temperature $T$ (b) at three different positions: (-.....) $x_{P_{1}}=1.5 \mathrm{H},(--) x_{P_{2}}=3.5 \mathrm{H}$, and $(-) x_{P_{3}}=5.5 \mathrm{H}$. Dotted lines denote the overlap region.

plied near the shocks seems efficient since the robustness is obtained without smoothing shocks or removing turbulence generated by the shock-boundary-layer interactions.

Mean velocity and temperature profiles are plotted at three different locations, $x_{P_{1}}=1.5 \mathrm{H}, x_{P_{2}}=3.5 \mathrm{H}$, and $x_{P_{3}}$ $=5.5 \mathrm{H}$, in Fig. 10. After each compression, the boundary layer thickens, the turbulence develops and as a result, the temperature increases near the wall due to viscous effects. The boundary layer thickness, defined as the location at which $U=0.99 U_{\max }$, is estimated as follows for the three positions, $\delta_{P_{1}} \simeq 2.8 \times 10^{-3} \mathrm{~m}, \delta_{P_{2}} \simeq 3.6 \times 10^{-3} \mathrm{~m}$, and $\delta_{P_{3}}$ $\simeq 6.4 \times 10^{-3} \mathrm{~m}$, and the friction velocities have also been computed, $u_{f, P_{1}} \simeq 9.8 \mathrm{~m} / \mathrm{s}, \quad u_{f, P_{2}} \simeq 9.4 \mathrm{~m} / \mathrm{s}$, and $u_{f, P_{3}}$ $\simeq 8.4 \mathrm{~m} / \mathrm{s}$. These friction velocities are certainly underestimated since the turbulent boundary layer is under-resolved. Further investigations are needed but this point is out of the scope of the present study.

The transverse turbulence intensities are given in Fig. 11 for the three positions $P_{i}$. The shapes of the curves are reasonable and compare qualitatively well with results of litera-
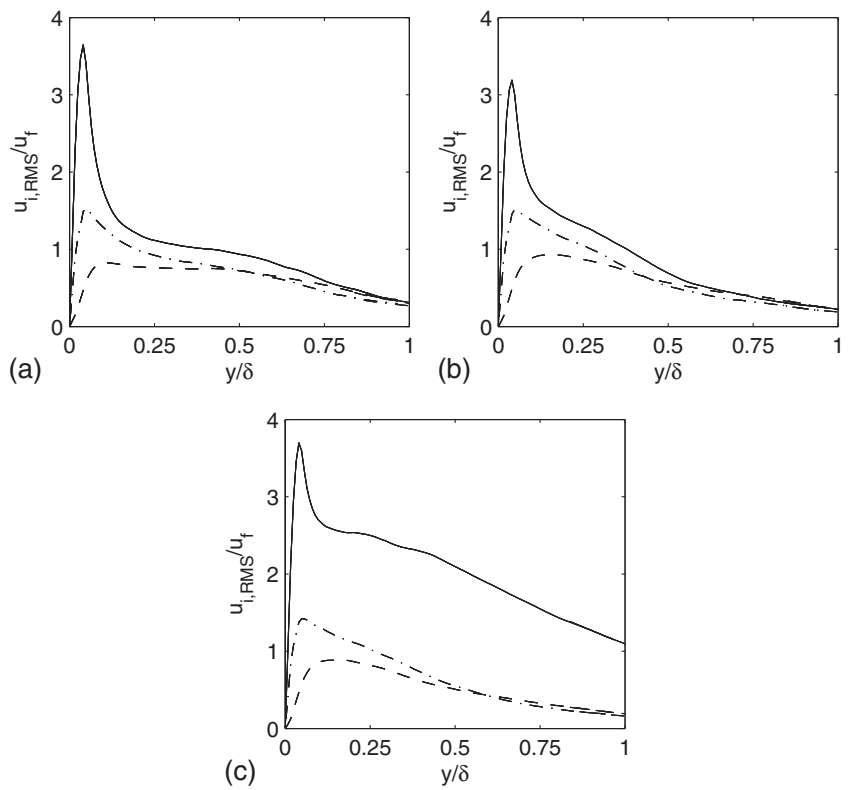

FIG. 11. Pressure ratio $\tau=0.15$, case R1. Transverse profiles of $(-) u^{\prime}$, $(--) v^{\prime}$, and (-...) $w^{\prime}$ at three different positions: (a) $x_{P_{1}}=1.5 H$, (b) $x_{P_{2}}=3.5 \mathrm{H}$, and (c) $x_{P_{3}}=5.5 \mathrm{H}$. 

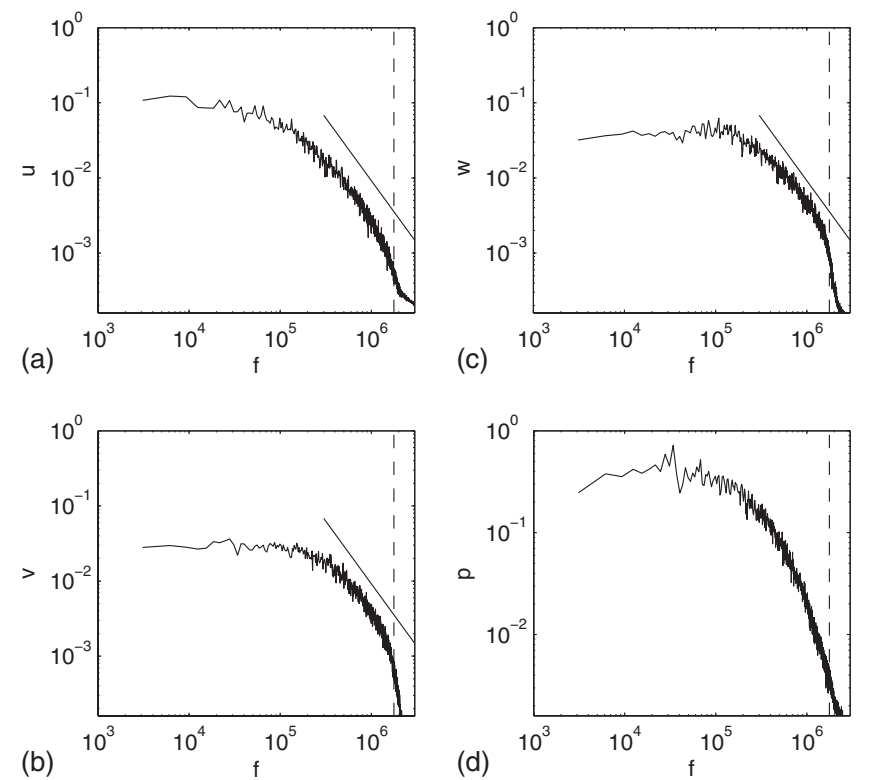

FIG. 12. Pressure ratio $\tau=0.15$, case R1. Frequency spectra at $(x, y)$ $=\left(3.5 H, 15 \Delta y^{+}\right)$for the three velocity components and for the pressure. The dashed line designates the cutoff frequency of the algorithm, estimated using Taylor's hypothesis, and the solid line represents Kolmogorov's 5/3-law.

ture found for free equilibrium boundary layers. At $P_{1}$, the peak of $u^{\prime}$ is overestimated compared to the boundary layer of Spalart, ${ }^{26}$ indicating that the boundary layer is still transitional. At position $P_{2}$, this peak is reduced indicating the presence of a more turbulent boundary layer. At position $P_{3}$, $u^{\prime}$ is entirely overestimated probably due to the variation of the bulk velocity caused by large shock motions in the axial direction that can be observed. Spectra of the velocity components and of the pressure computed at the position $(x, y)$ $=\left(3.5 H, 15 \Delta y^{+}\right)$are shown in Fig. 12. Despite the small length of the computational domain in the spanwise direction, which corresponds to approximately $6 \%$ of the channel length $L$, the spectrum of the spanwise component of the velocity indicates that the turbulent flow is also properly developed in the $z$-direction.

\section{TRANSONIC FLOW AT MODERATE PRESSURE RATIO}

For moderate pressure ratios, the flow is transonic in the main channel. Regime B associated with base-pressure oscillations is then in competition with regime $\mathrm{C}$, characterized by a steady asymmetrical flow pattern. As already mentioned in Sec. I, the two regimes coexist and the switch between these two states is driven by an hysteresis cycle, refer to Fig.
2. Four simulations with pressure ratios $\tau=0.30, \tau=0.31, \tau$ $=0.32$, and $\tau=0.35$ have been carried out, denoted by R2 $-\mathrm{R} 5$ in Table I. The grid spacings are summarized in Table III. The total number of grid points is $5.1 \times 10^{6}$. All the cases have been computed using $N_{\text {procs }}=32$ processors.

\section{A. Numerical results}

As highlighted in the experiments of Meier et al., ${ }^{3}$ a key parameter to identify the symmetrical and the asymmetrical flow patterns is the base pressure $p_{w}$ at the two upstream corners of the channel. Computed values obtained for the four computations are reported in Fig. 13, and a remarkable agreement is found with the measurements in the same geometrical configuration. The upper branch of the hysteresis loop, as well as the switch from the asymmetrical to the symmetrical flow regime, is correctly captured by the simulations. Note that for the computation at $\tau=0.32$, two slightly different values of the base pressure exist due to a weak inclination of the normal shock. The average gives the base pressure value corresponding to the symmetrical flow regime. Regimes $\mathrm{B}$ and $\mathrm{C}$ are presented in the following by considering cases R3 and R4 as illustration. The two additional simulations R2 and R5 have been performed to endorse numerical results, as shown in Fig. 13.

\section{Symmetrical base-pressure oscillations (regime B)}

Schlieren pictures distributed over 1 cycle of the basepressure oscillations are reported in Figs. 14 and 15 for case R3 at $\tau=0.31$. A general view of the flow features is first given. The flow pattern presents a normal shock interacting with the attached jet by a bifurcating or lambda structure on the lower and on the upper walls. This is a classical picture of a shock wave-boundary layer interaction. The pressure jump across the normal shock is transmitted into the boundary layer. Below the sonic line near the wall, the flow is subsonic and the pressure rise imposed by the shock produces a thickening of the boundary layer. In the present case, the front leg of the bifurcation corresponds to the oblique shock wave that is generated by the expansion waves generated at the nozzle exit and deflected by the channel walls. Downstream of the trailing leg, a supersonic tongue develops and the supersonic layers remain attached to the upper and lower walls. The flow downstream the normal shock is subsonic in the duct. In the vortex sheet layers separating the supersonic near wall layers and the subsonic flow in the channel, 2D instability rolls first develop, and these instabilities then give rise to turbulent 3D structures further downstream, near the duct outflow.

TABLE III. Characteristics of nozzle, expansion duct, and reservoir grid for cases R2-R5. The length scales are given in wall units $y^{+}=y u_{f} / \nu_{w}$, and the wall friction velocity and viscosity at the wall of case R1 are used; see Table II.

\begin{tabular}{lrrrrrrr}
\hline \hline & $N_{x}$ & $N_{y}$ & $N_{z}$ & $\Delta x_{\min }^{+}$ & $\Delta y_{\min }^{+}$ & $\Delta z_{\min }^{+}$ & $N_{\text {procs }}$ \\
\hline Nozzle & 29 & 49 & 31 & 42.1 & 28.1 & 42.1 & 1 \\
Expansion duct (central) & 510 & 161 & 31 & 42.1 & 28.1 & 42.1 & 15 \\
Reservoir & 203 & 398 & 31 & 42.1 & 28.1 & 42.1 & 16 \\
\hline \hline
\end{tabular}




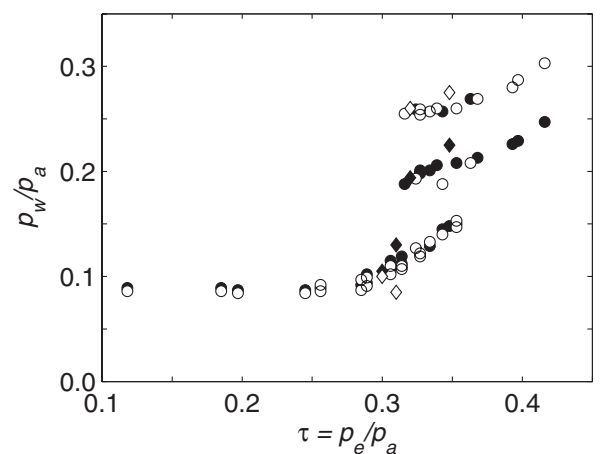

FIG. 13. Experimental data (Ref. 3) of the normalized mean base pressure $p_{w} / p_{a}$ as a function of the pressure ratio $\tau$ for cases R2-R5, see also Fig. 2, and the present simulations: $(\diamond)$ upper wall and $(\diamond)$ lower wall.

The base-pressure oscillation cycle can then be described as follows. For pictures (a)-(j) in Figs. 14 and 15, the supersonic jet is attached to the walls, and the normal shock in the channel gets stronger whereas the base pressure decreases. The shock-cell structures inside the supersonic layers are fully developed near the wall, and are clearly visible in Fig. 14(f), which could represent one end of the oscillation cycle. A stronger shock implies a higher downstream pressure, a larger section filled by the normal shock wave, and the supersonic layers near the wall become smaller. As the pressure increases downstream the shock, the configuration is not stable and the shock moves upstream. Then, as shown in Fig. 14(k), the shock is detached which allows a feedback between the downstream flow and the dead zone in the upstream corners where the base pressure $p_{w}$ is monitored. As this base pressure rises, the supersonic jet is contracted, the cross section of the shock decreases, and the flow is accelerated downstream in the duct, see Fig. 14(o). As a result, the flow entrainment decreases the base pressure, and a new normal shock is formed between Fig. 14(p) and Fig. 14(a).

The oscillation frequency is estimated as $f \simeq 350 \mathrm{~Hz}$ by the Fourier transform of the pressure signal $p_{w}(t)$, and can be compared to the value $f \simeq 373 \mathrm{~Hz}$ given by Meier $e t a l .{ }^{3}$ for the symmetrical base-oscillation frequency at $\tau=0.348$. Oscillations are weak and irregular, and no coupling with acoustic modes of the channel is observed in this configuration. Such a coupling is rather found for a shorter duct length $L$, as discussed in Sec. V.

\section{Asymmetrical steady flow (regime C)}

Another state can be reached by the flow as mentioned before, and the corresponding flow pattern obtained for case R4 at $\tau=0.32$ is shown in Fig. 16 as illustration of regime C. The steady flow is asymmetrical and is entirely separated from the lower duct wall. The isosurfaces of vorticity illustrate how the jet cross section is initially preserved, and how it begins to break up and mix more efficiently at the middle of the first shock cell. The jet reattaches after the end of the second shock cell. On the lower wall, regularly spaced strong perturbations traveling downstream can be observed, and a reversed flow is also found.

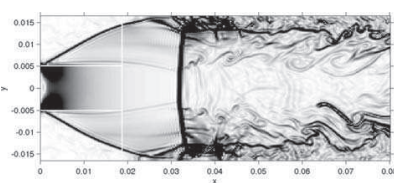

(a)

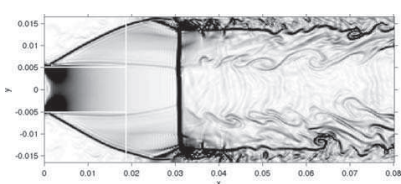

(b)

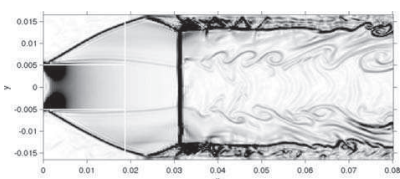

(c)

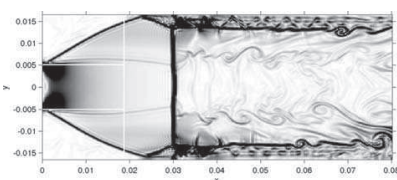

(d)

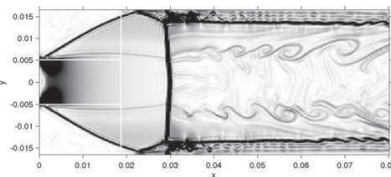

(e)

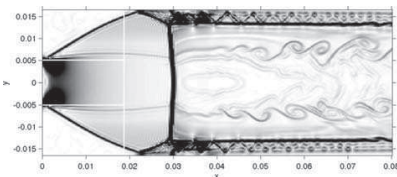

(f)

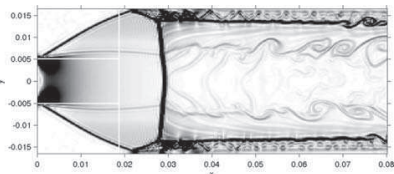

(g)

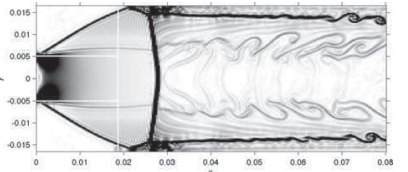

(h)

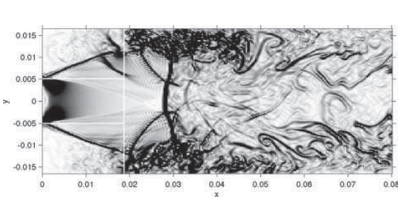

(I)

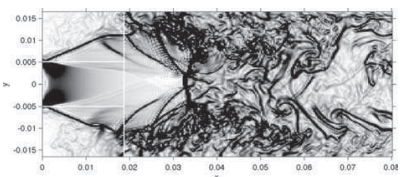

(m)

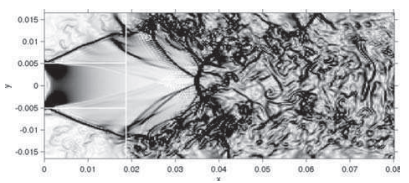

(n)

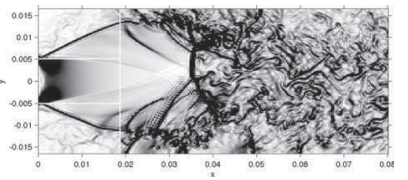

(0)

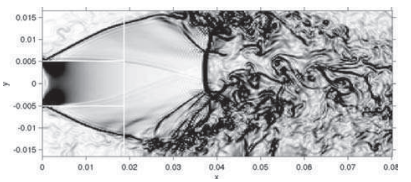

(p)

FIG. 14. Pressure ratio $\tau=0.31$, case R3. Schlieren-like pictures $0 \leq|\nabla \rho|$ $\leq 80 \mathrm{~kg} \mathrm{~m}^{-4}$ around the nozzle exit in the middle $x-y$ plane. Snapshots over a period of the base-pressure oscillations.

Figure 13 reveals the excellent agreement of the computed base pressures with the upper branch of the measurements. No aeroacoustic coupling is detected by the present simulations when the flow is asymmetrical. This is consistent with the experiments that do not exhibit acoustic feedback when the asymmetric transonic flow is established in the duct. Dominance of the asymmetric flow pattern in the hys- 

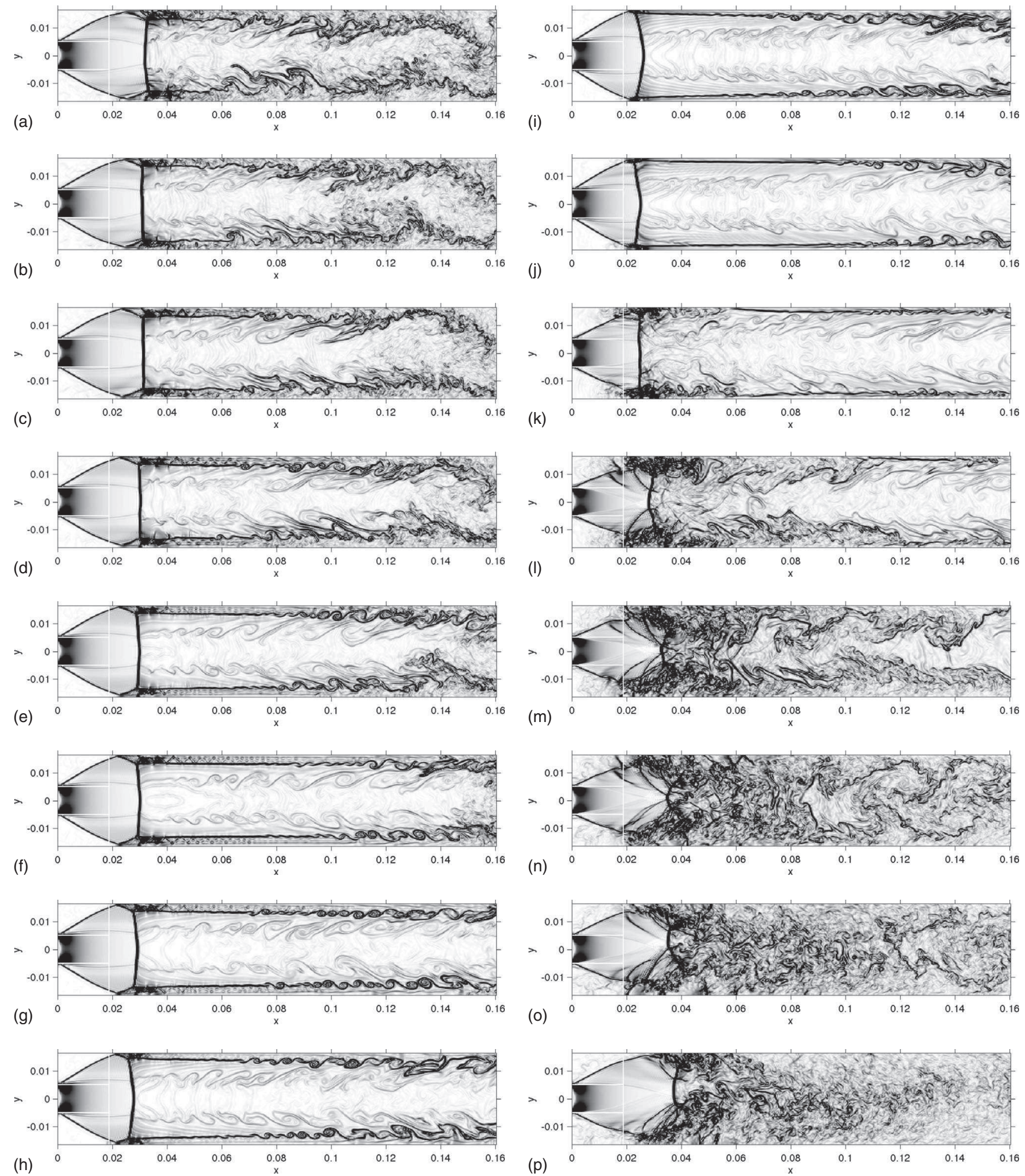

FIG. 15. Pressure ratio $\tau=0.31$, case R3. Schlieren-like pictures $0 \leq|\nabla \rho| \leq 80 \mathrm{~kg} \mathrm{~m}^{-4}$ of the whole channel duct in the middle $x-y$ plane. Snapshots over a period of the base-pressure oscillations.

teresis cycle could be attributed to the periodic boundary conditions used in the spanwise direction. The pressure in the upper and lower corner regions cannot be kept in balance as it would be the case when the lateral walls are present. Therefore the jet destabilizes and attaches to one duct side more easily.
Some turbulence statistics are given in Fig. 17 for the turbulent kinetic energy and the main turbulent shear-stress component. A high turbulent kinetic energy production is found along the upper and lower shear layers. On the attached side, turbulent production is endorsed by the presence of the wall. On the lower wall, the turbulent energy has 


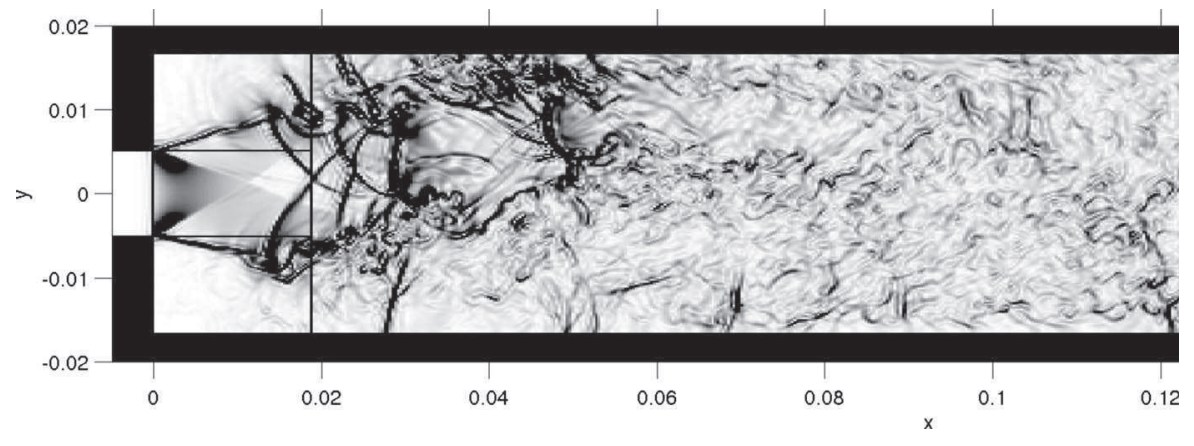

FIG. 16. Pressure ratio $\tau=0.32$, case R4. Schlieren-like view $0 \leq|\nabla \rho|$ $\leq 200 \mathrm{~kg} \mathrm{~m}^{-4}$ of the main channel on the middle $x-y$ plane.

reached its maximum downstream the first shock and drops to a constant value up to the end of the duct. This indicates a transition to a fully turbulent flow.

\section{B. Influence of the shock-capturing scheme}

As discussed before and illustrated in Fig. 13, the regime followed by the turbulent supersonic flow results from shock interactions with the turbulent boundary layers. Numerically, accuracy of the numerical solution is potentially damaged by the application of the adaptive nonlinear filtering, and this point is emphasized in the present section. As observed for the validation test cases performed before this study, ${ }^{27}$ the numerical dissipation introduced in the shock region can be minimized by the use of a higher-order Jameson sensor. Two computations have been carried out for case R4, one using the classical Jameson filter in the formulation of Kim and Lee, ${ }^{16}$ and one using the modified detector based on a more selective filter, transfer functions of the two filters are provided in Appendix A.

Computed spectra of the velocity and of the pressure are reported in Fig. 18 as illustration. Using the classical detector leads to slightly lower amplitudes, especially for the velocity component $w$ in the spanwise direction. This might be an indication that the transition to a turbulent state of the shear layer might be retarded due to higher dissipation introduced by the low order shock-capturing filter. Development of an efficient shock-capturing scheme combined with a lowdissipation and low-dispersion algorithm in computational aeroacoustics remains however a challenging problem. ${ }^{28}$
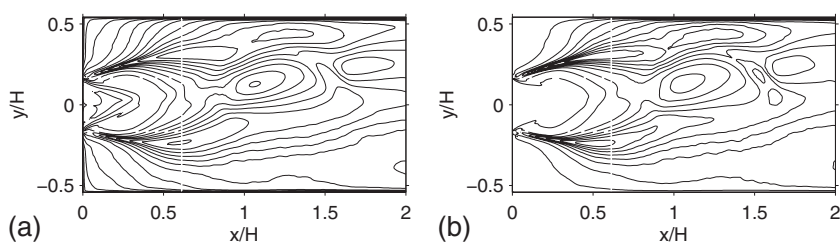

FIG. 17. Pressure ratio $\tau=0.32$, case R4. (a) Turbulent kinetic energy, 12 isocontours are plotted ranging from $0 \leq\left\langle u_{i}^{\prime \prime} u_{i}^{\prime \prime}\right\rangle / 2 \leq 25 \mathrm{~m}^{2} \mathrm{~s}^{-2}$. (b) Turbulent shear stress, ten isocontours are plotted ranging from $0 \leq\left|\left\langle u^{\prime \prime} v^{\prime \prime}\right\rangle / \bar{\rho}\right|$ $\leq 100 \mathrm{~m}^{2} \mathrm{~s}^{-2}$.

\section{SELF-EXCITED TRANSONIC FLOW OSCILLATIONS}

As discussed in Sec. I, a coupling between base-pressure oscillations and duct acoustic resonances can occur, and is sometimes observed experimentally ${ }^{2,3}$ for a given geometry. As reported by Meier et al., ${ }^{3}$ short ducts with small $h / H$ fail to oscillate whereas longer ducts fall in the asymmetrical flow regime $\mathrm{C}$. The self-excited mechanism is only observed for relative short ducts with area ratio such as $\phi=h / H$ $\geq 0.3$. In the present section, a more favorable configuration is therefore studied with a shorter length $L$ and a pressure ratio of $\tau=0.41$, refer to case R6 in Table I for the geometrical parameters. As a result of this choice, the Reynolds number is slightly decreased, $\operatorname{Re}_{h} \simeq 1.3 \times 10^{5}$, the shorter duct length leads to higher longitudinal mode frequencies, and thus to a reduction in the computation time. Moreover, the symmetrical flow regime $\mathrm{B}$ is preferred for this aspect ratio $h / H$. The pressure in the corner $p_{w}$ measured $^{3}$ as a function of the outflow pressure $p_{e}$ is displayed in Fig. 19. The hys-
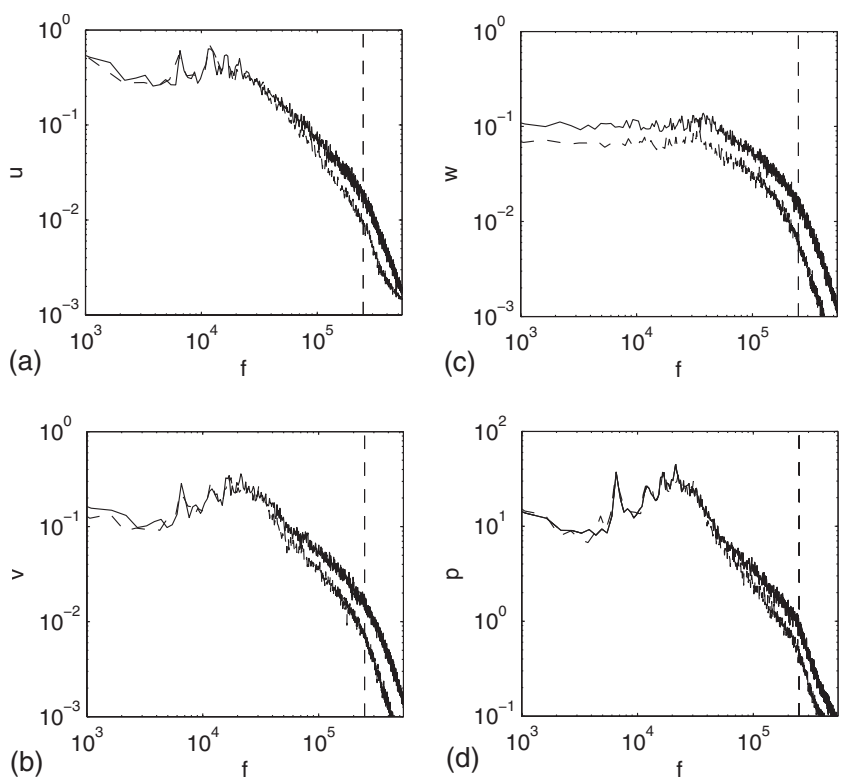

FIG. 18. Pressure ratio $\tau=0.32$, case R4. Frequency spectra at $(x, y)$ $=\left(3.5 H, 15 \Delta y^{+}\right)$for the velocity components and for the pressure, using $(---)$ the Jameson sensor and $(-)$ the modified Jameson sensor based on 11-point selective filter. The vertical dashed line designates the cutoff frequency of the algorithm, estimated by using Taylor's hypothesis. 


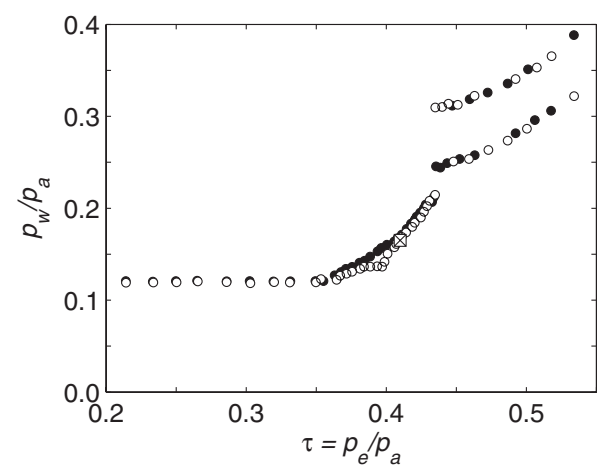

FIG. 19. $\tau=0.41$, case R6. Normalized mean base pressure measured (Ref. $3)$ as a function of the pressure ratio $\tau,(\bigcirc)$ upper wall and $(\bullet)$ lower wall. Present simulations, $(\square)$ upper wall and $(\times)$ lower wall.

teresis region is narrower than for the previous cases R2-R5, as shown in Fig. 13.

A new computational grid has been built using approximately the same number of mesh points as in Sec. IV, that is $5.1 \times 10^{6}$, and is distributed over $N_{\text {procs }}=165$ processors. Detail regarding the main overset meshes is provided in Table IV.

\section{A. A model for the aerodynamic field}

A Schlieren-like view of the whole computational domain is shown in Fig. 20. A normal shock wave can be observed in the upstream part of the duct and the flow remains subsonic downstream in the main duct, before reaching the plenum chamber in which the pressure is imposed at $p_{e}$ on the boundaries. A sponge layer is implemented at the duct exit to dissipate vortical structures without generating spurious sound. The acoustic radiation at the outflow of the duct is also visible in the picture and leaves the computational domain without spurious reflections.

The mean flow field is found symmetrical. The computed time averaged static pressure distributions along the upper and lower duct walls are given in Fig. 21(a). After the expansion, the compression zone indicates the location of the strong normal shock. Further downstream, the static pressure on the upper and the lower ducts converges to a value that is close to the downstream pressure $p_{e}=0.41 p_{a}$. The computed mean pressure in the upper and lower corners is $p_{w}$ $\simeq 0.165 p_{a}$ in excellent agreement with the measurements, as reported in Fig. 19. A relationship between the pressure ratio $p_{w} / p_{a}$ and the Mach number $M_{e}$ downstream the normal shock can be established using one dimensional compressible flow equations,

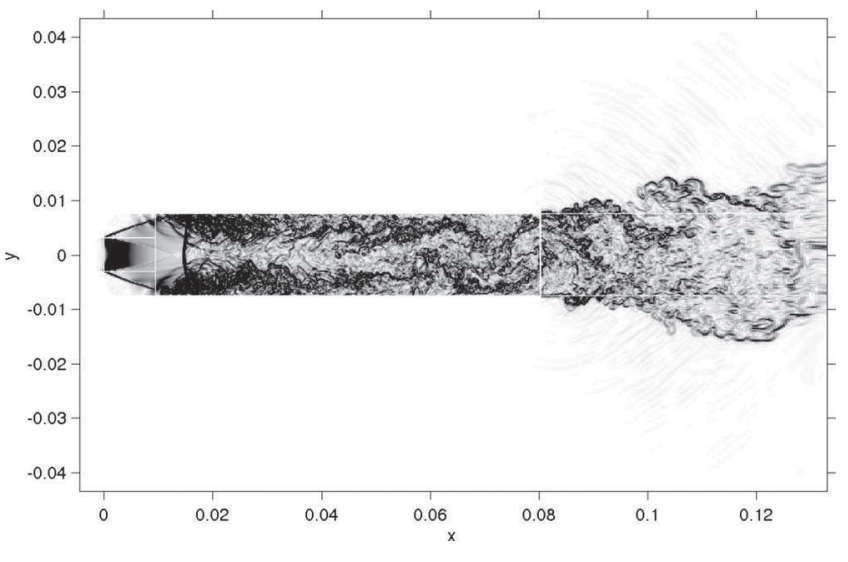

FIG. 20. Schlieren-like view $0 \leq|\nabla \rho| \leq 80 \mathrm{~kg} \mathrm{~m}^{-4}$ in the middle $x$-y plane of the whole computational domain including the reservoir.

$$
\begin{array}{r}
\frac{1-\phi}{\phi} \frac{p_{w}}{p_{a}}+(\gamma+1)\left(\frac{2}{\gamma+1}\right)^{\gamma /(\gamma-1)} \\
-\left(\frac{2}{\gamma+1}\right)^{(\gamma+1) /(2(\gamma-1))} \frac{1+\gamma M_{e}^{2}}{M_{e} \sqrt{1+\frac{\gamma-1}{2} M_{e}^{2}}}=0,
\end{array}
$$

where $\gamma$ is the ratio of specific heats, see Appendix B. For the computed value of the mean base pressure $p_{w}$, Eq. (1) yields a Mach number of $M_{e} \simeq 0.51$, as shown in Fig. 26, and this value is in good agreement with the computed Mach number along the center line $y=0$ of the channel, as displayed in Fig. 21(b).

\section{B. A model for the frequency locking of base-pressure oscillations}

Schlieren pictures distributed over one base-pressure oscillation cycle are shown in Fig. 22. Starting with picture (1), the base pressure decreases which induces a larger expansion of the exit supersonic jet, a stronger normal shock, and thus a higher pressure in the subsonic flow downstream. From pictures (d)-(i), an abrupt pressure adjustment occurs through the subsonic boundary layers, and the normal shock wave is recovered in picture ( $\mathrm{j}$ ), as described in Sec. IV A 1. However in the present case, the frequency of this selfexciting mechanism is imposed by the fundamental longitudinal acoustic resonance of the duct. The spectral peak of the wall pressure along the duct is plotted in Fig. 23 and this frequency is found to be $f \simeq 700 \mathrm{~Hz}$. The shape of the pressure amplitude is maximum at the nozzle exit and signifi-

TABLE IV. Characteristics of nozzle, expansion duct, and reservoir grid for case R6. The length scales are given in wall units $y^{+}=y u_{f} / \nu_{w}$, and the wall friction velocity and viscosity at the wall of case R1 are used; see Table II.

\begin{tabular}{lrrrrrrr}
\hline \hline & $N_{x}$ & $N_{y}$ & $N_{z}$ & $\Delta x_{\min }^{+}$ & $\Delta y_{\min }^{+}$ & $\Delta z_{\min }^{+}$ & $N_{\text {procs }}$ \\
\hline Nozzle & 29 & 65 & 31 & 21.5 & 12.9 & 21.5 & 6 \\
Expansion duct (central) & 510 & 161 & 31 & 21.5 & 12.9 & 21.5 & 77 \\
Reservoir & 203 & 398 & 31 & 21.5 & 12.9 & 21.5 & 82 \\
\hline \hline
\end{tabular}



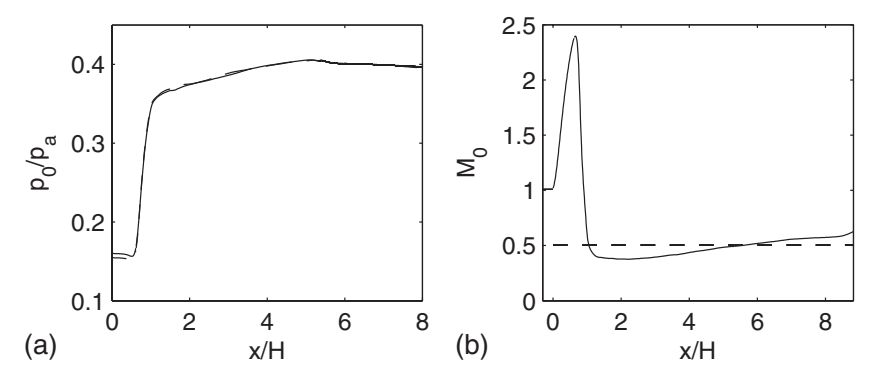

FIG. 21. $\tau=0.41$, case R6. (a) Computed mean pressure along the (-) upper and (- - ) lower wall. (b) Computed mean Mach number along the center line $y=0,(---)$ theoretical value downstream the shock provided by Eq. (1).

cantly diminishes when approaching the duct end, with a peak corresponding to the mean position of the normal shock wave.

In what follows, an estimation of the resonance frequency is proposed. In the presence of a uniform mean flow, the solution for the one-dimensional wave equation can be expressed as

$$
p^{\prime}=\left(A e^{i k^{+} x}+B e^{-i k^{-} x}\right) e^{-i \omega t}
$$

where $k^{+}=k_{0} /\left(1+M_{e}\right)$ and $k^{-}=k_{0} /\left(1-M_{e}\right)$ are the downstream and upstream wavenumbers, ${ }^{29} M_{e}$ is the Mach number, and $k_{0}=\omega / c_{e}$. For an ended-open tube, the boundary conditions are $\left.u^{\prime}\right|_{x=0}=0$ and $\left.p^{\prime}\right|_{x=L}=0$. Furthermore, in the presence of a mean flow, the boundary condition $u^{\prime}=0$ reads

$$
M_{e} \frac{1}{c_{e}} \frac{\partial p^{\prime}}{\partial t}+\left(M_{e}^{2}-1\right) \frac{\partial p^{\prime}}{\partial x}=0
$$

by using linearized Euler's equations. This expression replaces the classical Neumann boundary condition on the pressure for a medium at rest. Therefore, the eigenfrequencies are given by

$$
f_{r, n}=(2 n-1)\left(1-M_{e}^{2}\right) \frac{c_{e}}{4 L} .
$$

In the considered case, the mean Mach number $M_{e}$ is given by the subsonic flow behind the normal shock, estimated by Eq. (1), and $c_{e}$ corresponds to the mean sound velocity behind the shock. The upstream part of the duct can be treated as a closed end since a sonic flow is always present at the throat, and therefore no information can travel upstream through the nozzle. An end correction must be introduced ${ }^{29,30}$ at $x=L$, but only the no-mean flow case for a flanged circular duct is well established in literature. Following Ingard and Shingal, ${ }^{29}$ the effective duct length is then estimated by $L$ $+\delta L$ with $\delta L=0.8217(H / 2) /\left(1-M_{e}^{2}\right)$. The resonance frequency of the quarter-wave mode can thus be estimated as follows:

$$
f_{r, 0}=\left(1-M_{e}^{2}\right) \frac{c_{e}}{4 L_{\mathrm{eq}}}, \quad L_{\mathrm{eq}} \simeq L+0.8217 \times H / 2 .
$$

Using $M_{e} \simeq 0.51$ and $T_{e}=T_{a} /\left(1+(\gamma-1) / 2 \times M_{e}^{2}\right)$, one gets $c_{e} \simeq 334 \mathrm{~m} \mathrm{~s}^{-1}$, a value in agreement with the calculated mean flow, and $f_{r, 0} \simeq 721 \mathrm{~Hz}$ for the fundamental mode frequency. Although the value predicted by the model is in

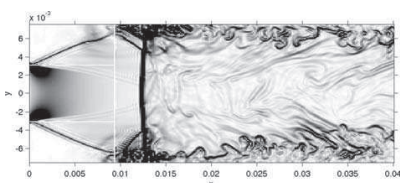

(a)

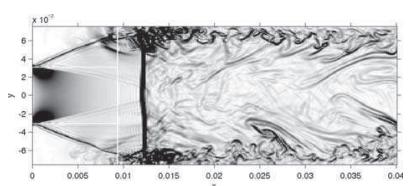

(b)

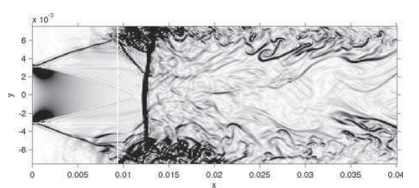

(c)

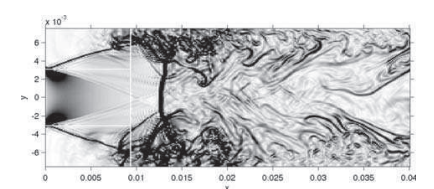

(d)

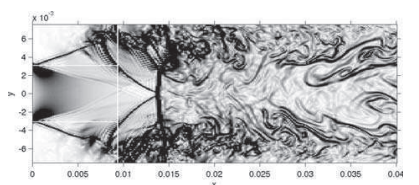

(e)

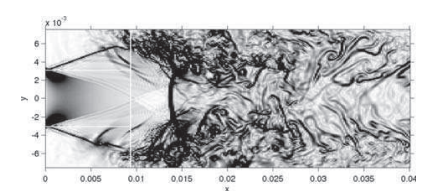

(f)

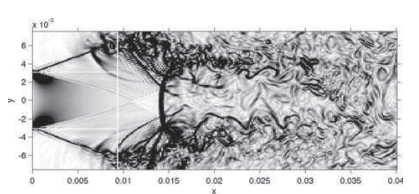

(g)

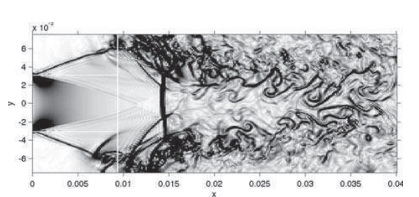

(h)

FIG. 22. Pressure ratio $\tau=0.41$, case R6. Schlieren pictures $|\nabla \rho|$ around the nozzle exit in the middle $x-y$ plane. Snapshots over a period of the selfexcited base-pressure oscillations.

agreement with the computed value, the importance of the impedance condition at $x=L$ has to be emphasize. In the present case, $k H=2 \pi f_{r, 0} H / c_{e} \simeq 0.23$, and therefore the condition $\left.p^{\prime}\right|_{x=L}=0$ seems reasonable. ${ }^{29}$ Periodic boundary conditions are used in the spanwise direction, and the resonance frequency is certainly modified with respect to the full scale 


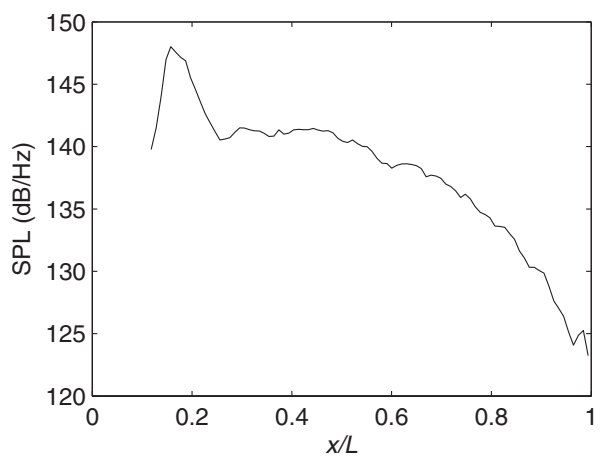

FIG. 23. Pressure ratio $\tau=0.41$, case R6. Computed sound pressure level of the wall pressure along the main channel for the frequency peak at $f$ $\simeq 700 \mathrm{~Hz}$ with a spectral resolution $\Delta f=50 \mathrm{~Hz}$.

experiment. The experimental value reported by Meier et al. ${ }^{3}$ is about $f \simeq 650 \mathrm{~Hz}$. In this simulation, and as observed in the experiments, the frequency selection of the base-pressure oscillations is imposed by the first longitudinal acoustic resonance of the channel. Moreover, the computed pressure level is quite high, about $148 \mathrm{~dB} / \mathrm{Hz}$ at this frequency.

\section{CONCLUSIONS}

In the present paper, the transonic flow passing a sudden enlargement of cross section has been studied by using largeeddy simulations based on an explicit relaxation filtering. The three main regimes found experimentally by Meier et $a l .{ }^{3}$ and Anderson et $a l^{2}{ }^{2}$ were recovered as a function of the pressure ratio between the exit pressure in the plenum chamber and the upstream ambient pressure. For low pressure ratios, the flow remains supersonic in all the test duct and consists of oblique shock waves reflected by the walls and interacting with the boundary layers. For a moderate pressure ratio, a transonic flow is found inside the main channel and two states can be reached. In one case, the exit supersonic jet is attached to one side and no strong pressure fluctuations are generated. In the other case, low-frequency base-pressure oscillations are produced by the normal shock motion, and a self-excited cycle has been reported in this study. For the six computed configurations, Schlieren visualizations as well as base-pressure ratios compare favorably with the measurements, indicating that the correct flow regime is well captured by the simulations. These simulations also show that the abrupt switch from symmetrical to asymmetrical flow pattern is well predicted and follows the hysteresis branch obtained for decreasing pressure ratios. The asymmetric flow pattern is the preferred configuration and this could be attributed to the periodic boundary used in the spanwise direction. No aeroacoustic coupling is observed for the asymmetrical flow pattern, in agreement with experiments.

Furthermore, a strong coupling between the first quarterwavelength resonance of the duct and the self-excited base pressure oscillations induced by the normal shock wave near the nozzle exit has been exhibited for one case, as also found in the experiments. A simple model has been revisited to support the present numerical results, and the computed resonance frequency is found in reasonable agreement with the predicted and the measured values. This kind of configura- tion must be avoided in pipe flow systems since the generated sound pressure levels are quite high. Note that this problem has motivated the development of the present study.

A new solver based on computational aeroacoustics algorithms has been developed to perform these simulations, including an additional nonlinear low-order filter applied to shock regions and an overset grid approach. This algorithm is able to reproduce the different flow regimes and the aeroacoustic couplings. High fidelity simulations of unsteady shock wave-boundary layer interactions occurring in confined transonic flows will however require additional efforts, as well as the simulation of the full 3D configuration in order to hope to reproduce the correct impedance conditions at the end of the channel.

\section{ACKNOWLEDGMENTS}

The authors would like to thank William Henshaw, from the Lawrence Livermore National Laboratory, for providing useful advice about the Overture library, and Professor Daniel Juvé, Ecole Centrale de Lyon, for helpful discussions.

\section{APPENDIX A: ADAPTIVE NONLINEAR FILTERING}

When the flow contains strong shocks, an additional numerical dissipation is introduced by using the adaptive nonlinear artificial dissipation model of Kim and Lee. ${ }^{16}$ These regions are identified using a Jameson-like sensor. Considering only the $x$-direction to simply algebra, the following procedure is applied on the pressure field:

$$
\Phi_{i}^{\xi}=\frac{\left|\sum_{r=-n}^{n} d_{r} p_{i+r}\right|}{\left|p_{i}-\sum_{r=-n}^{n} d_{r} p_{i+r}\right|},
$$

where $d_{r}$ are the coefficients of the chosen filter. The function $\Phi$ takes its maxima in regions containing shocks, and is very small in smooth regions. The classical Jameson detector ${ }^{31}$ based on a second-order Laplacian filter is retrieved by taking $n=1$. The Jameson detector has shown to be too sensitive to pressure fluctuations, and an excessive filtering of turbulent structures may occur, as observed by Ducros et al. ${ }^{32}$ for instance. Several approaches are possible to improve this shock-detection procedure in order to minimize the dissipation outside of the shocks and to preserve the generated acoustic field, as the use of the dilatation field ${ }^{28} \theta=\nabla \cdot \boldsymbol{u}$ rather than the pressure for the $\Phi$-function.

In the present study, the classical Laplacian has been replaced by a higher-order filter. Its transfer function in Fourier's space can be analyzed using a plane wave ansatz $\exp (-j k x)$ on a uniform grid of step size $\Delta x$, where $k$ is the wavenumber. It yields

$$
\hat{\Phi}_{i}^{\xi}(k \Delta x)=\frac{\left|\sum_{r=-n}^{n} d_{r} \exp (-j r k \Delta x)\right|}{\left|1-\sum_{r=-n}^{n} d_{r} \exp (-j r k \Delta x)\right|} .
$$

The sensor strength $\hat{\Phi}_{i}^{\xi}$ is displayed in Fig. 24 as a function of the normalized wavenumber $k \Delta x$ for different filtering operators, namely, the standard second-order $(n=1)$, fourthorder $(n=2)$, and tenth-order $(n=5)$ filters, and also the 11 point optimized filter ${ }^{10}(n=5)$. Using higher-order detectors leads to lower values in the low wavenumber range, whereas 


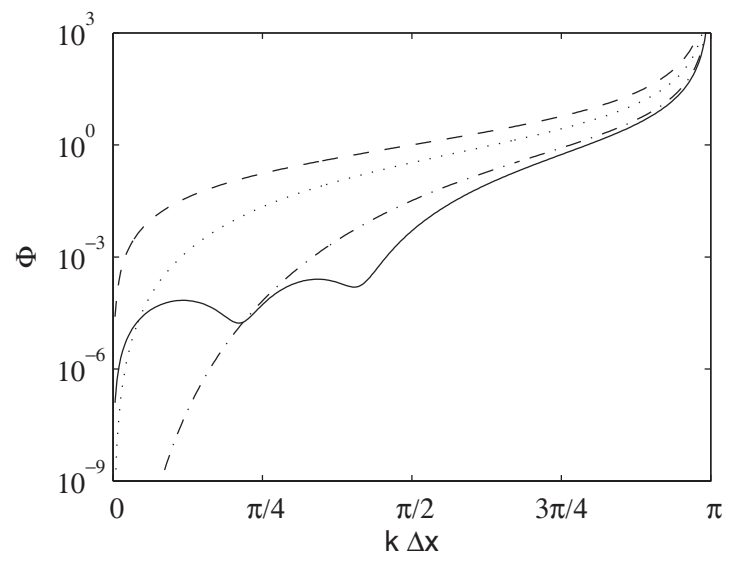

FIG. 24. Transfer function of the detector strength $\hat{\Phi}_{i}^{\xi}$ given by Eq. (A2) as a function of the normalized wavenumber $k \Delta x$. (- - ) Second-order filter (classical Jameson, $n=1) ;(\cdots \cdots \cdots)$ fifth-order filter $(n=2) ;(-\cdot-\cdot-)$ tenth-order filter $(n=5) ;(-)$ 11-point optimized filter $(n=5)$.

for the high frequencies, the detector behaves as the classical Jameson detector ensuring the shock-capturing property of the procedure. A similar approach was proposed by Lockard and Morris ${ }^{33}$ using a sixth-order filtering.

\section{APPENDIX B: DERIVATION OF THE RELATION FOR THE BASE PRESSURE}

In the experimental works, ${ }^{2,3}$ the relation between the pressure ratio $p_{w} / p_{a}$ and the Mach number $M_{e}$ downstream the normal shock is not reported. This relationship can be established using one-dimensional compressible flow equations. For steady, inviscid and adiabatic flow, and for a control volume fixed in space and bounded by a closed surface $S$, the integral conservation of mass, momentum, and total enthalpy $h_{t}$ can be written as

$$
\begin{aligned}
& \oint_{S} \rho \boldsymbol{u} \cdot \boldsymbol{n} d s=0, \\
& \oint_{S}(\rho \boldsymbol{u} \boldsymbol{u} \cdot \boldsymbol{n}+p \boldsymbol{n}) d s=0, \\
& \oint_{S} \rho h_{t} \boldsymbol{u} \cdot \boldsymbol{n} d s=0,
\end{aligned}
$$

where $\boldsymbol{n}$ is the outward pointing unit normal of $S$. By applying these relations to the control volume displayed in Fig. 25, the three shock equations or jump conditions are obtained,

$$
\begin{aligned}
& \rho_{h} u_{h} h=\rho_{e} u_{e} H, \\
& p_{w}(H-h)+\left(p_{h}+\rho u_{h}^{2}\right) h=\left(p_{e}+\rho u_{e}^{2}\right) H, \\
& \rho_{h} u_{h} h_{t h} h=\rho_{e} u_{e} h_{t e} H .
\end{aligned}
$$

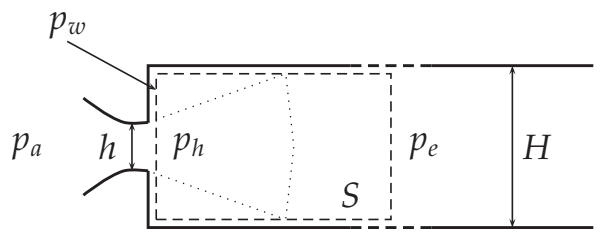

FIG. 25. Sketch of the control volume (dashed line) for the derivation of the jump equations, and of the supersonic flow (dotted line) just behind the sudden expansion.

Solving Eq. (B2) for the pressure $p_{w}$ and remembering that $\phi=h / H$, it follows that

$$
(1-\phi) \frac{p_{w}}{p_{a}}+\phi(\gamma+1)\left(\frac{2}{\gamma-1}\right)^{\gamma /(\gamma-1)}=\left(1+\gamma M_{e}^{2}\right) \frac{p_{e}}{p_{a}}
$$

for a perfect gas where use has been made of $M_{h}=1$ and $p_{a} / p_{h}=[(\gamma+1) / 2]^{\gamma /(\gamma-1)}$. Substituting Eq. (B1) into Eq. (B3), the temperature ratio $T_{h} / T_{e}$ can be expressed as

$$
\frac{T_{h}}{T_{e}}=\frac{2}{\gamma+1}\left(1+\frac{\gamma-1}{2} M_{e}^{2}\right) .
$$

Moreover, from Eq. (B1), it yields

$$
\frac{p_{h}}{p_{e}}=\frac{M_{e}}{\phi} \sqrt{\frac{T_{h}}{T_{e}}}
$$

Thus, substituting Eqs. (B5) and (B6) into Eq. (B4), the Rankine-Hugoniot relation relating the base pressure $p_{w}$ as a function of the downstream subsonic Mach number $M_{e}$ is obtained, corresponding to Eq. (1),

$$
\begin{aligned}
& \frac{1-\phi}{\phi} \frac{p_{w}}{p_{a}}+(\gamma+1)\left(\frac{2}{\gamma+1}\right)^{\gamma /(\gamma-1)} \\
&-\left(\frac{2}{\gamma+1}\right)^{(\gamma+1) /(2(\gamma-1))} \frac{1+\gamma M_{e}^{2}}{M_{e} \sqrt{1+\frac{\gamma-1}{2} M_{e}^{2}}}=0 .
\end{aligned}
$$

This relation is plotted in Fig. 26 for different nozzle aspect ratios $0.1 \leq \phi \leq 0.9$.

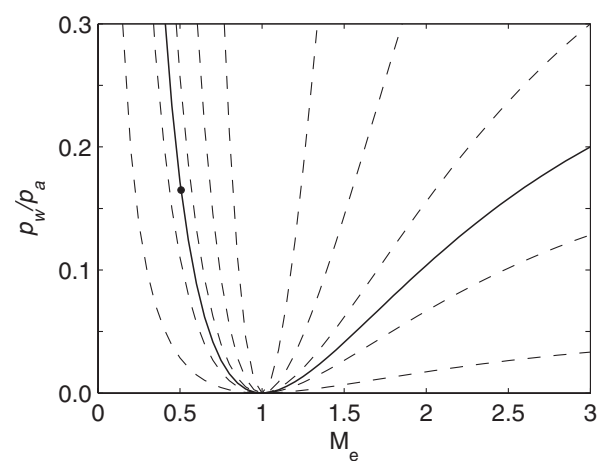

FIG. 26. Curves $p_{w}=p_{w}\left(M_{e}\right)$ for $\phi=0.1,0.3,0.5,0.7,0.9$ in dashed lines. Solid curve for $\phi=0.4$ corresponding to case R6 $(\bullet) p_{w} / p_{a} \simeq 0.165$ and $M_{e} \simeq 0.51$. 
${ }^{1}$ G. E. A. Meier, A. P. Szumowski, and W. C. Selerowicz, "Self-excited oscillations in internal transonic flows," Prog. Aerosp. Sci. 27, 145 (1990).

${ }^{2}$ J. S. Anderson, W. M. Jungowski, W. J. Hiller, and G. E. A. Meier, "Flow oscillations in a duct with a rectangular cross-section," J. Fluid Mech. 79, 769 (1977).

${ }^{3}$ G. E. A. Meier, G. Grabitz, W. M. Jungowski, K. J. Witczak, and J. S. Anderson, "Oscillations of the supersonic flow downstream of an abrupt increase in duct cross-section," Mitteilung aus dem Max-Planck-Institut fuer Stroemungsforschung und der Aerodynamischen Versuchsanstalt $\mathbf{6 5}$, 1 (1978).

${ }^{4}$ G. E. A. Meier, G. Grabitz, W. M. Jungowski, K. J. Witczak, and J. S. Anderson, "Oscillations of the supersonic flow donwstream of an abrupt increase in duct cross section," AIAA J. 18, 394 (1980).

${ }^{5}$ T. Colonius and S. Lele, "Computational aeroacoustics: Progress on nonlinear problems on sound generation,” Prog. Aerosp. Sci. 40, 345 (2004).

${ }^{6}$ C. K. W. Tam, "Computational aeroacoustics: An overview of challenges and applications," Int. J. Comput. Fluid Dyn. 18, 547 (2004).

${ }^{7}$ P. Lafon and J.P. Devos, "Numerical prediction of instabilities in transonic internal flows using an Euler TVD code," AAIA Paper No. 1993-72, 1993.

${ }^{8}$ M. R. Visbal and D. V. Gaitonde, "On the use of higher-order finitedifference schemes on curvilinear and deforming meshes," J. Comput. Phys. 181, 155 (2002).

${ }^{9}$ O. Marsden, C. Bogey, and C. Bailly, "High-order curvilinear simulations of flows around non-Cartesian bodies," J. Comput. Acoust. 13, 731 (2005).

${ }^{10}$ C. Bogey and C. Bailly, "A family of low dispersive and low dissipative explicit schemes for noise computation," J. Comput. Phys. 194, 194 (2004).

${ }^{11} \mathrm{C}$. Bogey and C. Bailly, "On the application of explicit spatial filtering to the variables or fluxes of linear equations," J. Comput. Phys. 225, 1211 (2007).

${ }^{12} \mathrm{C}$. Bogey and C. Bailly, "Large eddy simulations of transitional round jets: Influence of the Reynolds number on flow development and energy dissipation," Phys. Fluids 18, 065101 (2006).

${ }^{13} \mathrm{C}$. Bogey and C. Bailly, "Turbulence and energy budget in a selfpreserving round jet: Direct evaluation using large-eddy simulation," J. Fluid Mech. 627, 129 (2009).

${ }^{14}$ J. Berland, C. Bogey, and C. Bailly, "Numerical study of screech generation in a planar supersonic jet," Phys. Fluids 19, 075105 (2007).

${ }^{15}$ O. Marsden, C. Bogey, and C. Bailly, "Direct noise computation of the turbulent flow around a zero-incidence airfoil," AIAA J. 46, 874 (2008).

${ }^{16}$ J. W. Kim and D. J. Lee, "Adaptative nonlinear artificial dissipation model for computational aeroacoustics," AIAA J. 39, 810 (2001).
${ }^{17}$ J. A. Benek, J. L. Steger, and F. C. Dougherty, "A flexible grid embedding technique with applications to the Euler equations," AIAA Paper No. 831944, 1983.

${ }^{18}$ J. W. Delfs, "An overlapped grid technique for high resolution CAA schemes for complex geometries," AIAA Paper No. 2001-2199, 2001.

${ }^{19}$ F. Daude, T. Emmert, F. Crouzet, F. Lafon, and C. Bailly, "A high-order algorithm for compressible LES in CAA applications," AIAA Paper No. 2008-3049, 2008.

${ }^{20}$ S. E. Sherer and J. N. Scott, "High-order compact finite-difference methods on general overset grids," J. Comput. Phys. 210, 459 (2005).

${ }^{21}$ C. K. W. Tam and Z. Dong, "Radiation and outflow boundary conditions for direct computation of acoustic and flow disturbances in a nonuniform mean flow," J. Comput. Acoust. 4, 175 (1996).

${ }^{22} \mathrm{C}$. Bogey and C. Bailly, "Three-dimensional non-reflective boundary conditions for acoustic simulations: Far field formulation and validation test cases," Acta Acust. 88, 463 (2002).

${ }^{23}$ Y. Andreopoulos, J. H. Agui, and G. Briassulis, "Shock wave-turbulence interactions," Annu. Rev. Fluid Mech. 32, 309 (2000).

${ }^{24}$ W. M. Jungowski, "Investigation of flow pattern, boundary conditions and oscillation mechanism in a compressible flow through sudden enlargement of a duct," Ph.D. thesis, Warsaw University of Technology, 1968.

${ }^{25}$ A. J. Smits and J. P. Dussauge, Turbulent Shear Layers in Supersonic Flows, 2nd ed. (Springer, New York, 2006).

${ }^{26} \mathrm{P}$. R. Spalart, "Direct simulation of a turbulent boundary layer up to $R_{\theta}$ =1410," J. Fluid Mech. 187, 61 (1988).

${ }^{27}$ T. Emmert, P. Lafon, and C. Bailly, "Numerical study of aeroacoustic oscillations in a transonic flow donwstream a sudden duct enlargement," AIAA Paper No. 2006-2555, 2006.

${ }^{28}$ C. Bogey, N. De Cacqueray, and C. Bailly, "A shock-capturing methodology based on adaptative spatial filtering for high-order non-linear computations," J. Comput. Phys. 228, 1447 (2009).

${ }^{29}$ U. Ingard and V. Singhal, "Effect of flow on the acoustic resonances of an open-ended duct," J. Acoust. Soc. Am. 58, 788 (1975).

${ }^{30}$ Y. Nomura, I. Yamamura, and S. Inawashiro, "On the acoustic radiation from a flanged circular pipe,” J. Phys. Soc. Jpn. 15, 510 (1960).

${ }^{31}$ A. Jameson, W. Schmidt, and E. Turkel, "Numerical solutions of the Euler equations by finite volume methods using Runge-Kutta time-stepping schemes," AIAA Paper No. 81-1259, 1981.

${ }^{32}$ F. Ducros, V. Ferrand, F. Nicoud, C. Weber, D. Darracq, D. Gacherieu, and T. Poinsot, "Large eddy simulation of the shock/turbulence interaction," J. Comput. Phys. 152, 517 (1999).

${ }^{33}$ D. P. Lockard and P. J. Morris, "A parallel implementation of a computational aeroacoustic algorithm for airfoil noise," J. Comput. Acoust. 5, 337 (1997). 